\title{
Die Wahl der Strategie für den Eintritt auf asiatische Märkte durch brandenburgische und Berliner Unternehmen
}

\author{
Prof. Dr. Bernd Wonneberger, Dipl.-Psych.Hans Könecke, Dr. Rudolf Swat
}

\section{Einleitung}

Die Untersuchungen zur Wahl der Strategie zum Eintritt auf asiatische Märkte durch brandenburgische und Berliner Unternehmen fuihrten die Autoren im Rahmen des Forschungsprojektes „Markterschließung in Ost- und Südostasien für kleine und mittlere Unternehmen KMU in betrieblicher Kooperation und Erprobung praxisnaher Methoden der Fachhochschulausbildung" durch, welches von Oktober 1996 bis November 1997 an der Technischen Fachhochschule Wildau durchgefuihrt wurde.

Ausgangspunkt dieses Forschungsprojektes war die Feststellung, daß der Anteil der asiatischen Länder am Außenhandel Brandenburgs und Berlins im Jahre 1995 jeweils etwa 10 Prozent betragen hatte (ausfuihrlich dazu WONNEBERGER u. a. 1997a). Dieser im Vergleich zu europäischen Ländern geringe Anteil legt die Vermutung nahe, daß das schnell wachsende wirtschaftliche Potential der Länder Asiens zu wenig als Marktchance durch deutsche Unternehmen wahrgenommen wird. Eine Aussage, die letztlich für die Unternehmen in allen 16 Bundesländern gilt, wie entsprechende Veröffentlichungen des Bundesministerium für Wirtschaft BMWi und des Deutschen Industrie- und Handelstages DIHT zeigen.

Für die verstärkte Erschließung asiatischer Wachstumsmärkte durch die Unternehmen der Bundesländer Brandenburg und Berlin bestand eine Zielstellung unseres Forschungsprojektes darin, die wesentlichen Chancen und Probleme bei der Markterschließung empirisch zu erfassen, um daraus ggf. Lösungsvorschläge für die Intensivierung des Handels mit asiatischen Partnern ableiten zu können.

Getreu dem Motto „Viele Wege führen nach Asien“ müssen sicherlich, je nach den Voraussetzungen im Unternehmen selbst, dem Wirtschaftszweig sowie den Vertriebsformen und dem jeweiligen Marktpotential in den einzelnen Ländern Asiens, ganz unterschiedliche Wege der Markterschließung gegangen resp. Strategien gewählt werden. In den folgenden Analysen wollen wir der Frage nachgehen, welche Faktoren die Wahl der Strategie zum Eintritt auf asiatische Märkte durch Unternehmen in Berlin und Brandenburg bestimmen. Dabei gilt es auch zu überprüfen, inwieweit die Unternehmen in Brandenburg und Berlin sieben Jahre nach der deutschen Wiedervereinigung jene Strategien praktizieren, die von Unternehmen in anderen Bundesländern unter marktwirtschaftlichen Bedingungen seit Jahrzehnten angewandt werden. Die in den 80er Jahren gestellten Fragen zu der Internationalisierungsstrategie der kleinen und mittleren Unternehmen sind somit neu an die Berliner und brandenburgischen Unternehmen zu stellen.
Die Autoren wollen im folgenden zeigen, wie diese Unternehmen, unter veränderten gesellschaftlichen und weltwirtschaftlichen Bedingungen, ihre Strategie zur Erschließung asiatischer Märkte wählen.

\section{Problempräzisierung und Untersuchungsdesign}

Die aus der Literatur bekannten empirischen Analysen zur Wahl der Strategien zur Internationalisierung eines KMU basieren überwiegend auf Unternehmensvariablen, welche die Größe und Finanzkraft eines Unternehmens kennzeichnen (Mitarbeiterzahl, Umsatz, Rendite, und Eigenkapitalquote). Eine exakte empirische Bestimmung der Faktoren Eigenkapital und Rendite erscheint den Autoren schwierig, da viele Unternehmen hierzu freiwillig keine Angaben machen (vgl. BEUTEL 1988). Weitere Unternehmensvariablen, beispielsweise der Fertigwarenbestand oder der Auftragsbestand, wurden - bei Unternehmen aus den alten Bundesländern sowie Japan, Südafrika, Finnland und Österreich - von den Autoren (BEUTEL 1988, KÖGLMAYR 1990, MÜLLER 1991) mit divergierenden Ergebnissen untersucht; ein stabiler Einfluß dieser Faktoren auf die Strategiewahl konnte nicht nachgewiesen werden. Für unsere Untersuchung der Strategien zur Erschließung asiatischer Märkte durch Unternehmen aus Berlin und Brandenburg haben wir deshalb die Faktoren Eigenkapitalquote, Rendite, Fertigwarenbestand und Auftragsbestand nicht erneut erhoben.

In den zitierten Studien aus den 80er Jahren wurden unserer Meinung nach die Zusammenhänge zwischen den von Unternehmen präferierten Strategien und der Marktstellung (Branche, Kunden, Vermarktungsformen) des Unternehmens auf dem deutschen Heimatmarkt nicht ausreichend detailliert analysiert. So wäre beispielsweise zu erwarten, daß eine Vermarktung über deutsche Handelspartner zur Wahl einer anderen internationalen Strategie führen könnte, als bei einer direkten Vermarktung in Deutschland vom Hersteller zum Kunden.

In der von uns durchgeführten Erhebung wurden Berliner und brandenburgische KMU nach ihrer Bewertung bestimmter Strategien zur Erschließung asiatischer Märkte (Zielgröße) und nach Wirtschaftszweig, Branche, Kunden, Vermarktungsform, Ländermärkte, Mitarbeiterzahl, Jahresumsatz sowie Exportanteil (der klärenden Größen, Bestimmungsfaktoren) befragt.

Einige Autoren (BEUTEL 1988, KÖGLMAYR 1990, MÜLLER 1991) haben in ihren Studien aus den 80er Jahren eine Fülle von Daten in leitfadengestützten Interviews 
erhoben. Den genannten Autoren gelang es, in ihren Studien in Deutschland 78 bzw. 103 Interviewpartner zu gewinnen. Für statistisch gesicherte Analysen sind Stichproben dieses Umfangs zu klein. Da wir eine maximale Gewinnung möglichst vieler Unternehmensdaten anstrebten, schien den Autoren eine Befragung in Form einer schriftliche Erhebung am besten geeignet, da sich in unseren Vorstudien folgende Probleme mit anderen Erhebungsarten zeigten:

a) Telefonische Interviews: Zunächst einmal war es sehr zeitaufwendig, Geschäftsführer oder Manager überhaupt telefonisch zu erreichen; hierfür waren vielfach drei bis fünf Anrufe notwendig. Während der Telefonate zeigte sich dann, daß die Dauer eines telefonischen Interviews möglichst 15 Minuten nicht überschreiten sollte, weil ansonsten die Geduld und Gesprächsbereitschaft der Interviewpartner stark nachließ. Ein detaillierterer Interviewleitfaden konnte in vielen Fällen innerhalb von 15 Minuten nicht komplett durchgearbeitet werden.

b) Persönliche Interviews: Für die Terminvereinbarung waren vielfach drei bis fünf Anrufe notwendig. Bedingt durch die Größe des Flächenstaates Brandenburg, entstand hier ein hoher Aufwand durch die Fahrtzeiten (vgl. HENNING/SCHIEWE 1997), so daß zu vertretbaren Kosten die Bildung einer großen Stichprobe nicht möglich geworden wäre.

Somit entschlossen wir uns zur Entwicklung eines neunseitigen, überwiegend standardisierten Fragebogens, welcher (nach erfolgreichem Pretest und geringfuigigen Änderungen) bei unserer schriftlichen Befragung im November 1996 an 2.000 kleine und mittelständische Unternehmen (10 bis 999 Mitarbeiter), die Mitglied der Industrie- und Handelskammern Berlin, Cottbus, Frankfurt (Oder) und Potsdam sind, verschickt wurde.

Die Gesamtzahl der kleinen und mittelständischen Unternehmen als Mitglieder der genannten Industrie- und Handelskammern betrug im November 1996 ca. 2.400 Unternehmen in ausgewählten Wirtschaftszweigen (vgl. dazu Kapitel 3). Von den 2.000 versandten Fragebögen erhielten wir bis zum 10. Dezember 1996 einen Rücklauf von 269 auswertbaren Fragebögen.

\section{Struktur der Stichprobe von branden- burgischen und Berliner Unternehmen}

\subsection{Wirtschaftszweige und Stammsitz}

Der Wirtschaftszweig der von uns befragten Unternehmen läßt sich nach der Standard Industrial Classification (SIC-Code) im Mitgliederverzeichnis der IHK eindeutig empirisch bestimmen. Abweichungen zwischen der (amtlichen) Klassifizierung der Unternehmen nach Wirtschaftszweigen und deren aktuellem Leistungsspektrum seien an dieser Stelle nur kurz erwähnt, jedoch nicht ausführlicher diskutiert (vgl. dazu HENNING und SCHIEWE, S. 66). Die Architektur- und Ingenieurbïros werden z. B. nur durch die Wahl der Rechtsform einer GmbH zu Mitgliedern der IHK; freiberuflich tätige Architekten und
Ingenieure werden dort nicht erfaßt. Die Tabelle 1 gibt einen Überblick iiber die Wirtschaftszweige der von uns befragten 269 Unternehmen.

\begin{tabular}{|l|r|r|c|}
\hline Wirtschaftszweig & SIC-Code & Anzahl & Prozent \\
\hline Papiergewerbe & 21 & 5 & 1,9 \\
Verlagsgewerbe & 22 & 20 & 7.4 \\
Chemische Industrie & 24 & 8 & 3,0 \\
Gummi- und Kunstwaren-Herstellung & 25 & 5 & 1.9 \\
Glas, Keramik, Baustoffe & 26 & 4 & 1,5 \\
Metallerzeugung/-bearbeitung & 27 & 5 & 1,9 \\
Herstellung von Metallerzeugnissen & 28 & 23 & 8.6 \\
Maschinenbau & 29 & 24 & 8,9 \\
Büromaschinen und DV-Geräte & 30 & 3 & 1,1 \\
Elektrotechnik & 31 & 17 & 6.3 \\
Rundfunk-, TV- und Nachrichtentechnik & 32 & 5 & 1,9 \\
Medizin, MSR, Optik & 33 & 15 & 5.6 \\
Bau von Kraftfahrzeugen & 34 & 4 & 1,5 \\
Sonstiger Fahrzeugbau & 35 & 3 & 1,1 \\
Recycling & 37 & 2 & 0,7 \\
Datenverarbeitung & 72 & 19 & 7.1 \\
Forschung & 73 & 1 & 0,4 \\
Architekten und Ingenieurbüros & 74 & 90 & 33,5 \\
ohne Angaben & & 16 & 5.9 \\
\hline Summe & & 269 & 100,0 \\
\hline
\end{tabular}

Tab. 1: Wirtschaftszweigstruktur der Gesamtheit der 269 Unternehmen

Der Stammsitz befand sich bei 164 der befragten Unternehmen im Bundesland Berlin, bei weiteren 73 im Bundesland Brandenburg; die anderen Unternehmen hatten ihren Stammsitz in anderen Bundesländern sowie eines in Südkorea; zehn Unternehmen machten keine Angaben zu ihrem Stammsitz.

\subsection{Branchen}

In der wirtschaftswissenschaftlichen Literatur wird der Begriff der Branche vielfach synonym verwendet zum Begriff des Wirtschaftszweiges. Die bereits in Abschnitt 3.1. genannte Klassifikation des SIC-Codes der Wirtschaftszweige basiert beim verarbeitenden Gewerbe in der Regel auf den Materialeigenschaften der produzierten und verarbeiteten Güter (Papiergewerbe, Chemische Industrie usw.).

Aus Sicht des Marketing sind weniger die Materialeigenschaften, sondern vielmehr die Funktionen der einzelnen Wirtschaftsgüter, also der Unterschied zwischen Konsum- und Investitionsguitern, bestimmend für die Strategie ihrer (internationalen) Vermarktung. Dies wurde bei der Konzeption des Fragebogens von den Autoren berüicksichtigt, um eine für die Problemstellung relevante Gruppierung der Unternehmen zu generieren. Mit der Frage „Unser Unternehmen zählt zur Branche ..." baten wir um eine Selbsteinstufung der Unternehmen als Hersteller von Investitionsguitern, Hersteller von Konsumgütern und Anbieter von Dienstleistungen. In Auswertung der von den Unternehmen gegebenen Antworten ergibt sich die in Abbildung 1 dargestellte Struktur der Stichprobe nach Branchen.

Wie in der Abbildung zu sehen, ist die Selbsteinstufung der KMU in die drei Branchen (Investitionsgüterher- 
steller, Konsumguiterhersteller; Anbieter von Dienstleistungen) nicht disjunkt. So gibt es insbesondere zwischen den Herstellern von Investitionsgütern und den Anbietern von Dienstleistern Mehrfachnennungen.

Für die weiteren Analysen wurde aus der originären, nicht disjunkten Gruppierung der Branchen (vgl. Abb.1) die in Tabelle 2 dargestellte disjunkte Gruppierung mit fünf Gruppen gebildet.

\begin{tabular}{|lcc|}
\hline Branchen (nach Gruppenbildung) & Anzahl & Prozent \\
\hline $\begin{array}{l}\text { Investitionsgiter-Hersteller ohne Angebot von } \\
\text { Dienstleistungen }\end{array}$ & 83 & 30,9 \\
$\begin{array}{l}\text { Konsumgüter-Hersteller ohne Angebot von } \\
\text { Dienstleistungen }\end{array}$ & 49 & 18,2 \\
reine Anbieter von Dienstleistungen & 92 & 34,2 \\
Hersteller von Investitions- oder Konsumgittern \\
mit dem Angebot von Dienstleistungen & 18 & 6,7 \\
keine Angaben & 27 & 10,0 \\
\hline Summe & 269 & 100,0 \\
\hline
\end{tabular}

Tab. 2: Verteilung der Unternehmen nach Branchen (disjunkte Gruppierung)

Auch in unserer Erhebung fanden wir vereinzelt technologieorientierte Unternehmen, deren Entwicklung bereits Kulicke unter dem Stichwort „Entstehung durch Härtung“ wie folgt beschrieben hat: Zunächst weist ein neugegründetes Unternehmen nicht die Merkmale eines Technologieunternehmens auf (z. B. Dienstleistungsunternehmen oder Anbieter von Low-Tech-Produkten). „Aber nach einigen Jahren des Bestehens führt ein Entwicklungsprojekt zu einem eigenen innovativen Produkt oder Verfah- ren für einen größeren Kundenkreis“ (KULICKE, S. 50). Bei der schriftlichen Befragung antworteten uns vor allem Investitionsgüteranbieter und Dienstleister, die am Export bzw. internationalen Marketing im allgemeinen, aber auch speziell in Richtung Asien Interesse haben. Wir nehmen an, daß gerade diese Unternehmen noch am Beginn ihrer Internationalisierung stehen.

\subsection{Kundentypen und Vermarktungsformen}

Nach den Kundentypen der Unternehmen fragten wir mit Frage 18: „Wir verkaufen unsere Produkte überwiegend an ... ". Die Nennungen hierzu sind in Tabelle 3 abgebildet, wobei überwiegend Mehrfachnennungen gegeben wurden. Die Spalte Anzahl Nennungen enthält die Anzahl der Nennungen des jeweiligen Kundentyps. Die Spalte exklusive Anzahl Nennungen enthält die Anzahl der Nennungen jener Unternehmen, die ausschließlich an einen Kundentyp ihre Produkte verkaufen.

\begin{tabular}{|l|c|c|}
\hline $\begin{array}{l}\text { Kundentypen } \\
\text { (Mehrfachnennungen waren möglich) }\end{array}$ & $\begin{array}{c}\text { Anzahl } \\
\text { Nennungen }\end{array}$ & $\begin{array}{c}\text { exklusive } \\
\text { Nennungen }\end{array}$ \\
\hline private Endverbraucher & 75 & 6 \\
gewerbliche Kunden & 216 & 89 \\
Gebietskörperschaften & 101 & 3 \\
(Bund/.änder/Gemeinden) & 115 & 5 \\
andere öffentliche Auftraggeber & 13 & 3 \\
sonstige Abnehmer & & \\
\hline
\end{tabular}

Tab. 3: Kundentypen der Unternehmen

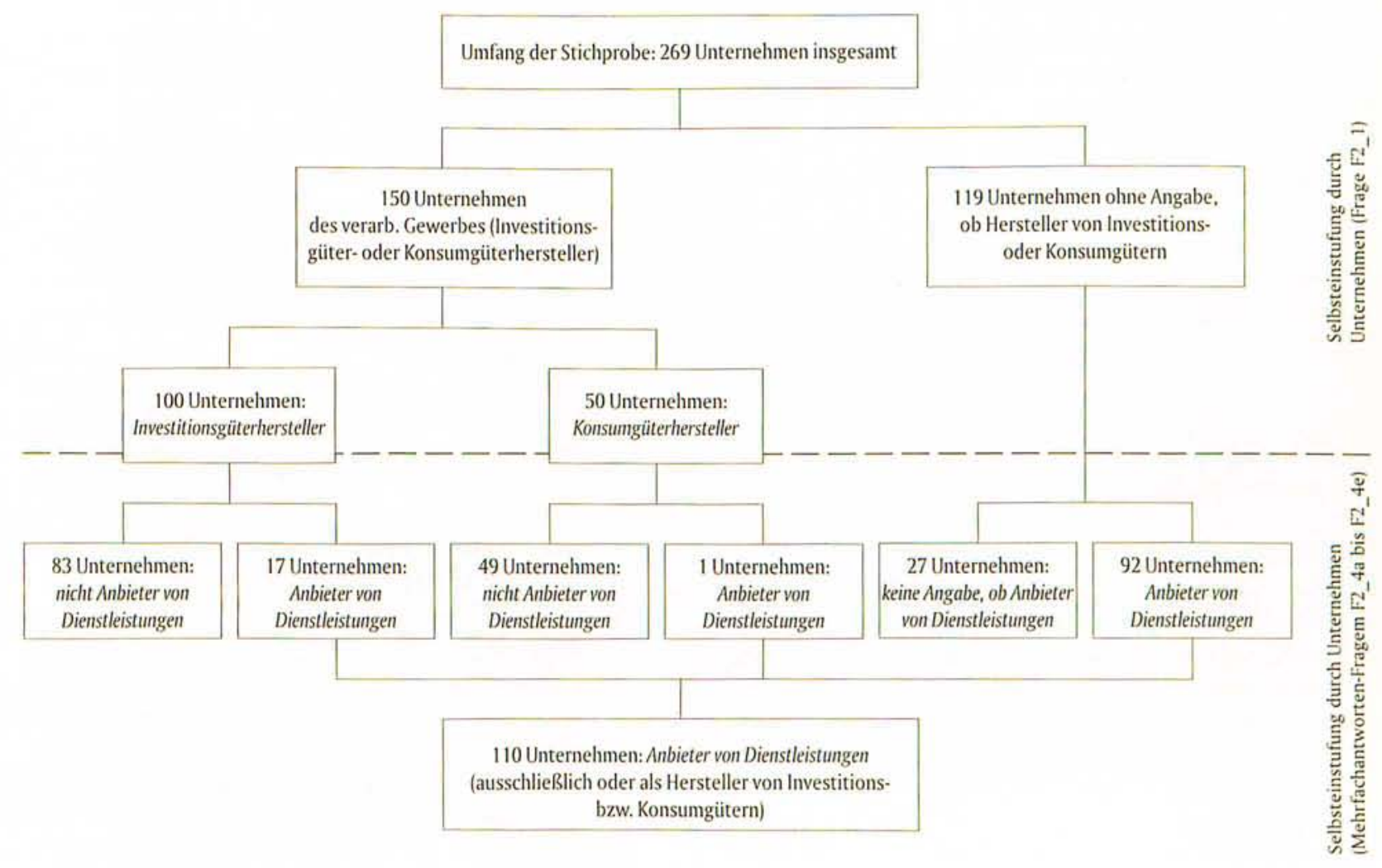


Wie vermarkten die befragten Unternehmen ihre Produkte und Dienstleistungen? Die (Mehrfach-)Antworten der Unternehmen zu dieser Frage sind in Tabelle 4 dargestellt, es gelten hierfür die gleichen Erläuterungen wie für Tabelle 3. Man sieht, daß bei den Unternehmen unseres Samples die direkte Vermarktung über eigene Vertriebswege im Vordergrund steht, was bei InvestitionsguiterHerstellern und Dienstleistern die Regel ist (vgl. BACKHAUS 1992, KLEINALTENKAMP und PLINKE 1995).

\begin{tabular}{|l|c|c|}
\hline $\begin{array}{l}\text { Vermarktungsformen } \\
\text { (Mehrfaclunenutungen waren möglich) }\end{array}$ & $\begin{array}{c}\text { Anzahl } \\
\text { Nennungen }\end{array}$ & $\begin{array}{c}\text { exklusive } \\
\text { Nennungen }\end{array}$ \\
\hline direkt & 236 & 187 \\
über Groß- und Einzelhandel & 55 & 18 \\
sonstige Formen & 26 & 3 \\
keine Angaben & 9 & - \\
\hline
\end{tabular}

Tab. 4: Vermarktungsformen der Unternehmen

Als Hypothesen für den weiteren Gang unserer Analyse nehmen wir an, daß die (branchenspezifischen) Erfahrungen der Unternehmen mit den Kundentypen und Vermarktungsformen auf ihrem Heimatmarkt einen bestimmenden Einfluß haben könnte auf die Wahl ihrer Strategien zur Erschließung asiatischer Märkte.

\section{Definition der verschiedenen Strategie- formen für den Eintritt auf asiatische Märkte}

In der Literatur zum Internationalen Marketing werden verschiedene Strategien genannt, die sich unterscheiden hinsichtlich des Kapitaleinsatzes und der Kontrollrechte für jenes Unternehmen, welches internationale Märkte erschließen möchte (vgl. STAHR 1993, S. 64). Die Kombination beider Merkmale führt zu der in Abbildung 2 dargestellten Gruppierung von insgesamt 10 einzelnen Strategien.

\begin{tabular}{|l|l|l|}
\hline & geringe Kontrolle & hohe Kontrolle \\
\hline $\begin{array}{l}\text { hoher } \\
\text { Kapitaleinsatz }\end{array}$ & $\begin{array}{l}\text { 1a) Joint Venture } \\
\text { 1b) Kontraktproduktion } \\
\text { 1c) Projektbeteiligung }\end{array}$ & $\begin{array}{l}\text { 2a) Produktions- oder } \\
\text { Montageunternehmen }\end{array}$ \\
\hline $\begin{array}{l}\text { geringer } \\
\text { Kapitaleinsatz }\end{array}$ & $\begin{array}{l}\text { 3a) Export über } \\
\text { Handelshäuser }\end{array}$ & $\begin{array}{l}\text { 4a) Export im Direktvertrieb } \\
\text { 4b) Technische Kooperations- } \\
\text { abkommen }\end{array}$ \\
& 3b) Franchising & 4c) Kontraktmanagement \\
\hline
\end{tabular}

Abb. 2: Gruppierung von Strategien im internationalen Marketing nach Kapitaleinsatz und Kontrollrechten

Die zehn einzelnen Strategien werden somit in vier Gruppen zusammengefaßt. Nachfolgend wollen wir diese Strategieformen in Anlehnung an Stahr kurz definieren, wobei das ausländische Unternehmen jeweils ein KMU aus Brandenburg wäre, welches mit unterschiedlichen lokalen Partnern oder autonom einen Markt beispielsweise in Asien erschließen möchte (vgl. STAHR 1993, S. 65-72).

\section{Strategien mit geringen Kontrollrechten und hohem Kapitaleinsatz}

a) „Bei einem Joint Venture beteiligen sich ein oder mehrere lokale Partner (aus dem Zielland; d.V.) und das aus- ländische Unternehmen am Kapital einer Produktionsunternehmung. Partnerschaftsunternehmen können in der Form der Mehrheits-, Gleichheits- oder Minoritätsbeteiligung gegrïndet werden" (STAHR, S. 70).

b) Bei der Kontraktproduktion übernimmt ein Partnerunternehmen im Zielland die Herstellung und den Vertrieb der Produkte. Der (deutsche) Partner stellt - beispielsweise im Rahmen von Lizenzverträgen die Produkt- und Verfahrenstechnologie (vgl. STAHR, S. 70f).

c) „Bei der Projektbeteiligung handelt es sich in erster Linie um die Lieferung und Erstellung einer schlüsselfertigen Produktionsanlage durch einen ausländischen Hersteller. Die Fabrikationsstätte wird Hauptbestandteil einer neu gegründeten Unternehmung (Projektgesellschaft). ... Die Beteiligung entspricht einem Teil des gesamten Kaufpreises" (STAHR, S. 71).

Strategien mit hohen Kontrollrechten und hohem Kapitaleinsatz

a) Hierunter verstehen wir die Errichtung eines zu $100 \%$ beherrschten Produktions- oder Montageunternehmens im jeweiligen Zielland (vgl. STAHR, S. 72).

b) Eine unternehmenseigene Vertriebsniederlassung im Ausland ist wirtschaftlich abhängig vom Mutterunternehmen, sie kann als „Profitcenter mit Sitz im Ausland bezeichnet werden“. Ihre juristische Selbstständigkeit oder Abhängigkeit bestimmt sich dagegen nach den jeweils geltenden nationalen Gesetzen im Zielland (vgl. STAHR, S. 71f).

\section{Strategien mit geringen Kontrollrechten und gerin- gem Kapitaleinsatz}

a) Beim Export über Handelshäuser stellt das Unternehmen seine Produkte her und verkauft diese an qualifizierte Exporteure, welche dann auf eigene Rechnung und eigenes Risiko die Produkte im Zielland vermarkten. Der Produzent hat keinen Einfluß mehr auf die Vermarktung seiner Produkte (vgl. STAHR, S. 67).

b) „Franchising beinhaltet in erster Linie die Abgabe ... von kaufmännischem Know-how (Marketing, Logistik u. a. m.) an einen Franchisenehmer im jeweiligen Zielland" (STAHR, S. 68).

\section{Strategien mit hohen Kontrollrechten und geringem Kapitaleinsatz}

a) Beim Export im Direktvertrieb verkauft der Hersteller seine Leistungen und Produkte direkt an die Verwender im Zielland (vgl. STAHR, S. 69).

b) „Technische Kooperationsabkommen werden häufig mit staatlichen Institutionen ... abgeschlossen. In der Regel sind sie eine Ergänzung zu Lieferverträgen im Anlagengeschäft und beinhalten den Verkauf von Dienstleistungen in den Bereichen Projektierung, Know-how-Transfer, Ausbildung und Hilfen bei der Inbetriebnahme der Anlagen" (STAHR, S. 69). Ein Beispiel hierfur ist die Fahrmeister-Ausbildung der Metro in Shanghai durch das Unternehmen ADTRANZ, ehemals AEG (vgl. FORSCHNER 1996).

c) „Beim Kontraktmanagement bringt der Partner im Zielland das Kapital und das ausländische Unterneh- 
men das notwendige Know-how und das Management ein. ... Neben den Managementfunktionen übernimmt das ausländische Unternehmen eine strenge Kontrolle über die Geschäftsaktivitäten (vor allem Qualitätskontrolle)" (STAHR, S. 68).

In einer ersten Auswertung der Ergebnisse unserer Erhebung wird zu prüfen sein, welchen Stellenwert diese Strategien für KMU aus Brandenburg und Berlin haben. Zweitens soll untersucht werden, ob die von Stahr gebildeten vier Strategiegruppen für die Berliner und brandenburgischen KMU empirisch bestätigt werden können. Drittens ist zu prüfen, ob durch die von uns im 3. Kapitel analysierte Struktur erklärt werden kann, warum bestimmte Unternehmen eine bestimmte Strategie oder ein Bündel von Strategien überdurchschnittlich hoch (oder gering) bewerteten.

\section{Bewertung und empirische Gruppierung der Strategien zur Erschließung asiatischer Märkłe}

\subsection{Die Bewertung der verschiedenen Strategien durch Berliner und brandenburgische KMU}

In der von uns durchgefuihrten Erhebung wurden den befragten Unternehmen acht Strategien für den Eintritt auf asiatische Märkte (vgl. Tabelle 5) zur Bewertung auf einer 6-Punkte-Skala vorgelegt. Die Numerierung der Strategien bezieht sich in der ersten Spalte auf die im vierten Kapitel dargestellte Abbildung 2, in der zweiten Spalte auf die Nummerierung im Fragebogen.

\begin{tabular}{|c|c|c|}
\hline $\begin{array}{l}\text { Nr. } \\
\text { Abb. } 2\end{array}$ & $\begin{array}{l}\mathrm{Nr} \text {. } \\
\mathrm{FB}\end{array}$ & Strategie \\
\hline 3a) & 1 & $\begin{array}{l}\text { Export über europäische Handelshäuser und } \\
\text {-kommissionäre }\end{array}$ \\
\hline 3a) & 2 & $\begin{array}{l}\text { Export über Handelshäuser und -kommissionäre im } \\
\text { Zielland }\end{array}$ \\
\hline 4b) & 3 & $\begin{array}{l}\text { Lizenz- und Technologietransfer-Verträge mit } \\
\text { ausländischen Partnern }\end{array}$ \\
\hline 3b) & 4 & Franchising \\
\hline 4c) & 5 & $\begin{array}{l}\text { Rahmenverträge über Absatz und Vertrieb (Kontrakt- } \\
\text { management) }\end{array}$ \\
\hline 2b) & 6 & Eigene Vertriebsorganisation im Zielland \\
\hline 1a) & 7 & $\begin{array}{l}\text { Beteiligung an ausländischen Unternehmen } \\
\text { (Joint Venture) }\end{array}$ \\
\hline 2a) & 8 & Direkt-Kapitalinvestition zu $100 \%$ \\
\hline
\end{tabular}

Tab. 5: Übersicht über die von den Unternehmen zu bewertenden Strategien

Sinnvoll erschien den Autoren bei der Konzeption des Fragebogens die differenzierte Bewertung des Exportes über Handelshäuser/-kommissionäre entweder in Europa oder einem asiatischen Zielland. Jede dieser beiden Strategien (aus der Gruppe geringer Kapitaleinsatz mit geringen Kontrollrechten) hat spezifische Vorund Nachteile in punkto Marktkenntnis der Exporteure, Fakturierung, Zahlungsabwicklung u. a. m. Von der ersten Strategiegruppe (hoher Kapitaleinsatz mit geringen Kontrollrechten) haben wir dagegen nur explizit das Joint Venture bewerten lassen. In unseren Vorstudien hatten wir festgestellt, daß dieser Begriff von der Wirt- schaftspresse wie von den Unternehmen, vielfach synonym gebraucht wird auch fuir die Kontraktproduktion und Projektbeteiligung. Die inhaltlichen Unterschiede zwischen den Strategien dieser Gruppe werden letztlich erst deutlich bei den konkreten Vertragsverhandlungen und -gestaltungen der jeweiligen Partner.

Auch nach dem direkten Vertrieb hatten wir im Fragebogen nicht explizit gefragt, da wir annahmen, daß diese Strategie in Asien, auf Märkten außerhalb Europas, für ein Unternehmen aus Brandenburg oder Berlin zu hohe logistische Aufwendungen bedeuten würde. Wir hatten stattdessen in einer offenen Frage nach sonstigen Strategien gefragt; hierbei gaben sechs der befragten Unternehmen den direkten Export als eine ihrer Strategien an. Eine Übersicht der sonstigen genannten Strategien geben wir in der Tabelle 6.

\begin{tabular}{|lr|}
\hline Strategien - sonstige Nennungen & Anzahl \\
\hline Direkter Vertrieb & 6 \\
Know-how - Verträge mit Kooperationspartnern & 1 \\
Neugründung mit Hilfe ausländischer Studenten & \\
(Absolventen deutscher Universitäten) & 1 \\
Direkte Tourismuswerbung für Europa - mit Berlin-Trip & 1 \\
Weltbank, Asiatische Bank, KfW & 1 \\
Verkauf von Ingenieur-Service & 1 \\
Vertrieb durch deutsche Mitarbeiter; Ausbildung ausländischer & 1 \\
Mitarbeiter: Gründung eines Unternehmens in der Region & 2 \\
Messebesuche - regelmäßig & 1 \\
über Multiplikator - Kunden suchen & \\
\hline
\end{tabular}

Tab. 6: Strategien - sonstige Nennungen

Interessant erscheinen den Autoren jene zwei Unternehmen, die die Ausbildung ausländischer Mitarbeiter als Kern einer Internationalisierungsstrategie nennen. Bei dieser Strategie ist das Problem der Gewinnung qualifizierter Mitarbeiter mit guten Kenntnissen der Zielmärkte vergleichsweise leicht zu lösen. Leider entzieht es sich unserer Kenntnis, wieweit auch brandenburgische Unternehmen diese Strategie nutzen; uns liegen keine Untersuchungen darüber vor, wieviele ausländische Mitarbeiter in brandenburgischen Unternehmen beschäftigt werden. Dieses Instrument der Aus- und Weiterbildung ausländischer Studenten, Diplomanden und Arbeitnehmer läßt sich zudem nur voll nutzen, wenn die in letzter Zeit wiederholt aufgetretene, offene Ausländerfeindlichkeit in Brandenburg deutlich zurückgeht.

Die sonstigen durch die Unternehmen genannten Strategien mögen an einigen Stellen für den Praktiker interessante Anregungen enthalten; wegen der geringen Anzahl ihrer Nennungen können sie jedoch in den weiteren Analysen nicht berüicksichtigt werden.

\begin{tabular}{|c|c|}
\hline $\begin{array}{c}\text { Anzahl der je Unternehmen } \\
\text { bewerteten Strategien }\end{array}$ & $\begin{array}{c}\text { Anzahl der } \\
\text { Unternehmen }\end{array}$ \\
\hline 1 & 82 \\
2 & 25 \\
3 & 16 \\
4 & 9 \\
5 & 11 \\
6 & 4 \\
7 & 11 \\
8 & 111 \\
\hline & Summe: \\
\hline
\end{tabular}

Tab. 7: Verteilung der Anzahl der je Unternehmen bewerteten Strategien 
Von den vorgegebenen acht Strategien wurde durch jedes der befragten 269 Unternehmen mindestens eine Strategie bewertet, in 111 Fällen wurden sogar alle acht Strategien bewertet. Im Durchschnitt wurden von einem Unternehmen vier bis fünf Strategien bewertet.

Die Ergebnisse der Bewertung der acht Strategien werden wir im folgenden darstellen, wobei jedoch noch einige Vorbemerkungen notwendig erscheinen zur Interpretation der in Tabelle 8 dargestellten Ergebnisse. $\mathrm{Zu}$ beachten ist dabei die Konstruktion der Skala. Die Unternehmen wurden durch die Frage „Unsere Strategien für den Eintritt auf die asiatischen Märkte wären ..." aufgefordert, aus ihrer Sicht die Wichtigkeit der jeweils vorgegebenen Strategie auf einer sechsstufigen Punkteskala zu bewerten. Die Randpunkte der Skala waren mit 1 für „sehr wichtig“ und 6 für „unwichtig“ vorgegeben. Nach der gegebenen Fragestellung und der vorgegebenen Skala ist eine eindeutige Interpretation der MissingFälle nicht möglich. Einerseits können Missing-Fälle interpretiert werden als „keine Bewertung der Strategie“" oder auch als „Beantwortung vergessen/Frage übersehen". Andererseits kann eine Nichtbewertung der betreffenden Strategie aber auch bedeuten, „diese Strategie wäre für das Unternehmen nicht relevant“, mit anderen Worten „völlig unwichtig“. Unterstellt man die letztgenannte Interpretationsvariante bei der Analyse des Antwortverhaltens der Unternehmen, erscheint es bei den in Tabelle 8 dargestellten Ergebnissen durchaus plausibel, die Mehrheit der Missing-Fälle so zu interpretieren, als ob die jeweilige Strategie als „völlig unwichtig" bewertet worden sei.

\begin{tabular}{|c|c|c|c|c|c|c|c|c|c|}
\hline \multirow[b]{2}{*}{ Strategie } & \multirow[b]{2}{*}{ valide } & \multicolumn{6}{|c|}{ Punktwerte - relative Häufigkeit in $\%$ der validen Falle } & \multirow{2}{*}{$\begin{array}{c}\text { mittlerer } \\
\text { Punkt- } \\
\text { wert }\end{array}$} & \multirow{2}{*}{$\begin{array}{l}\text { Mis- } \\
\text { sing }\end{array}$} \\
\hline & & 1 & 2 & 3 & 4 & 5 & 6 & & \\
\hline 1 & 152 & 14,5 & 22,4 & 15,1 & 7,9 & 14,5 & 25.7 & 3,625 & 117 \\
\hline 2 & 154 & 33,1 & 24,0 & 17,5 & 5,8 & 7,1 & 12,3 & 2,669 & 115 \\
\hline 3 & 147 & 16,3 & 24,5 & 19,0 & 8,8 & 7.5 & 23,8 & 3,381 & 122 \\
\hline 4 & 122 & 1,6 & 6,6 & 16.4 & 9,0 & 12,3 & 54,1 & 4,861 & 147 \\
\hline 5 & 149 & 17,4 & 31,5 & 18,8 & 6,0 & 9,4 & 16,8 & 3,087 & 120 \\
\hline 6 & 141 & 19,9 & 15,6 & 17,0 & 5,0 & 12,1 & 30,5 & 3,652 & 128 \\
\hline 7 & 148 & 8.8 & 20.9 & 18,9 & 12,2 & 16,9 & 22,3 & 3,742 & 121 \\
\hline 8 & 132 & 3,8 & 7,6 & 9,1 & 6,1 & 20,5 & 53,0 & 4,909 & 137 \\
\hline
\end{tabular}

Tab. 8: Bewertung der Strategien nach Punktwerten

Die in Tabelle 8 abgebildeten relativen Häufigkeiten und mittleren Punktwerte für die Bewertung der von den Autoren vorgegebenen Strategien zeigen, daß die einzelnen Strategien hinsichtlich ihrer Bedeutung aus Sicht der befragten Unternehmen unterschiedlich bewertet werden. Die drei am höchsten bewerteten Strategien sind nach dem mittleren Punktwert:

2) Export über Handelshäuser und -kommissionäre im Zielland $(2,669)$,

5) Rahmenverträge uiber Absatz und Vertrieb (Kontraktmanagement) $(3,087)$ und

3) Lizenz- und Technologietransferverträge mit ausländischen Partnern $(3,381)$.

Als deutlich weniger wichtige Strategien wurden mit einem mittleren Punktwert von über 4,5 bewertet:
4) Franchising $(4,861)$ und

8) Direkt-Kapitalinvestition zu $100 \%(4,909)$.

Hoch bewertet wurden aus der Sicht der befragten Unternehmen Strategien, die einen vergleichsweise geringen Kapitaleinsatz erfordern. Demgegenüber wird die kapitalintensive Strategie 8 (Direkt-Kapitalinvestition zu $100 \%$ ) als deutlich weniger wichtig bewertet.

Die nicht kapitalintensive Strategie 4 (Franchising) wird ebenfalls als weniger wichtig bewertet. Ein Erklärung fuir diese Bewertung ergibt sich aus der Branchenstruktur unserer Stichprobe, die überwiegend Anbieter von Investitionsguitern und von Dienstleistungen enthält. Die Strategie des Franchising wird dagegen bis dato überwiegend praktiziert durch Anbieter von Konsumguitern.

\subsection{Empirische Gruppierung der Strategien}

Im vorangegangenen Abschnitt wurde analysiert, welche der acht Strategien im Durchschnitt aller befragten Unternehmen als sehr wichtig und welche Strategien als weniger wichtig bzw. gar nicht wichtig bewertet wurden. Eine weitere wichtige Frage besteht darin, ob in der Gesamtheit der acht bewerteten Strategien eine Struktur besteht. Zur Beantwortung dieser Frage analysierten wir die acht durch brandenburgische und Berliner KMU bewerteten Strategien mit Hilfe der Verfahren der Faktorenanalyse.

In Anwendung der Hauptkomponentenmethode wurden nach dem Eigenwertkriterium drei Faktoren extrahiert (siehe Tabelle 9). Die ersten drei Faktoren erklären die Gesamtstreuung in der Bewertung der acht Strategien zu 62,6 Prozent.

\begin{tabular}{|l|c|c|c|c|c|c|c|c|}
\hline & \multicolumn{7}{|c|}{ Faktor } \\
\cline { 2 - 9 } & 1 & 2 & 3 & 4 & 5 & 6 & 7 & 8 \\
\hline Eigenwert & 2,14288 & 1,5497 & 1,31333 & 0,88713 & 0,67159 & 0,57181 & 0.49780 & 0,36575 \\
\hline $\begin{array}{l}\text { Anteil an der } \\
\text { Gesamt- } \\
\text { steuerung |0| }\end{array}$ & 26,8 & 19,4 & 16,4 & 11,1 & 8,4 & 7,1 & 6,2 & 4,6 \\
\hline $\begin{array}{l}\text { kumulierte } \\
\text { Anteile }\end{array}$ & 26,8 & 46,2 & 62,6 & 73,7 & 82,1 & 89,2 & 95,4 & 100,0 \\
\hline
\end{tabular}

Tab. 9: Eigenwerte der Faktoren und ihr Anteil an der Gesamtsteuerung (Hauptkomponentenmethode)

Die drei extrahierten Faktoren erklären die Streuung in der Bewertung der einzelnen Strategien unterschiedlich (siehe Tabelle 10). Der größte Erklärungsanteil ergibt sich mit rund 76 Prozent für die Strategie 4 (Franchising) und der geringste Erklärungsanteil mit rund 50 Prozent für die Strategie 3 (Lizenz- und Technologietransferverträge mit ausländischen Partnern).

\begin{tabular}{|c|c|c|c|c|c|c|c|c|}
\hline \multirow{2}{*}{} & \multicolumn{7}{|c|}{ Strategie } \\
\cline { 2 - 9 } & 1 & 2 & 3 & 4 & 5 & 6 & 7 & 8 \\
\hline Kommunalität & 0.6204 & 0.7235 & 0,4922 & 0.7572 & 0.6357 & 0.6003 & 0.6098 & 0,566 \\
\hline
\end{tabular}

Tab. 10: Kommunalitäten für die acht Strategievariablen bei drei Faktoren (Hauptkomponentenmethode)

Um die Interpretationsfähigkeit der Faktorlösung zu verbessern, wurden die drei Faktoren nach der VarimaxMethode rotiert. Im Ergebnis ergab sich die in Tabelle 11 
dargestellte Einfachstruktur. Jede der acht Strategien ist nur einmal auf einen der drei Faktoren hochgeladen.

Der Faktor 1 wird vor allem durch drei Strategien bestimmt:

- Joint Venture (hohe Kapitalintensität/geringe Kontrolle),

- eigene Vertriebsorganisation im Zielland (hohe Kapitalintensität/hohe Kontrolle) und

- Direkt-Kapitalinvestitionen zu $100 \%$ (hohe Kapitalintensität/hohe Kontrolle).

Der Faktor 1 repräsentiert somit die kapitalintensiven Strategien mit unterschiedlichen Kontrollrechten.

Der zweite Faktor wird vor allem durch den Export über Handelshäuser bestimmt - Strategien mit geringer Kapitalintensität und geringen Kontrollrechten:

- Export über Handelshäuser im Zielland (geringe Kapitalintensität/geringe Kontrolle),

- Export über europäische Handelshäuser (geringe Kapitalintensität/geringe Kontrolle).

Auf den Faktor 3 sind drei Strategien hoch geladen:

- Franchising (geringe Kapitalintensität/geringe Kontrolle),

- Lizenz- und Technologietransferverträge (geringe Kapitalintensität/hohe Kontrolle) und

- Kontraktmanagement (geringe Kapitalintensität/hohe Kontrolle).

Im dritten Faktor dominieren somit vor allem Strategien mit geringer Kapitalintensität und unterschiedlichen Kontrollrechten.

\begin{tabular}{|c|c|c|c|c|}
\hline $\mathrm{Nr}$. & Strategie & Faktor 1 & Faktor 2 & Faktor 3 \\
\hline 7 & Joint Venture & $|.77757|$ &., 05750 & .04361 \\
\hline 6 & eigene Vertriebsorganisation im Zielland & .76545 & -.11404 &,- 03768 \\
\hline 8 & Direkt-Kapitalinvestition zu $100 \%$ & .74826 &, 08138 &,- 01083 \\
\hline 2 & Export über Handelshäuser im Zielland &,- 04019 & $\lceil .83283$ & -.16825 \\
\hline 1 & Export über europäische Handelshäuser & $\cdot, 26812$ & .73730 &, 07006 \\
\hline 4 & Franchising &., 08644 & .02960 & $\Gamma, 86538$ \\
\hline 3 & Lizenz- und Technologietransferverträge & .03913 & .32272 & $\mid .62173$ \\
\hline 5 & Kontraktmanagement & .08573 & .50200 & 1,61349 \\
\hline
\end{tabular}

Tab. 11: Sortierte Faktorladungsmatrix für 3 Faktoren (Hauptkomponentenmethode) nach der Varimax-Rotation

Die faktoranalytische Auswertung der Erhebungsergebnisse bestätigt die in Abschnitt 4 vorgestellte Gruppierung der Strategien nach ihrer Kapitalintensität. Die brandenburgischen und Berliner KMU unterscheiden in der Bewertung der für sie wichtigen Strategien vor allem zwischen kapitalintensiven und nicht-kapitalintensiven Strategien. Die Unterscheidung zwischen Strategien mit geringen bzw. hohen Kontrollrechten für das Unternehmen wurde durch die empirischen Ergebnisse dagegen nicht eindeutig bestätigt.

\section{Bestimmende Fakłoren für die Bewertung der Strategien}

Aus den bisherigen Darlegungen ergab sich, daß die befragten Berliner und brandenburgischen KMU die acht Strategien hinsichtlich ihrer Bedeutung für das eigene Unternehmen unterschiedlich bewerten. In diesem
Zusammenhang stellt sich die Frage, ob zwischen bestimmten ökonomischen, das Unternehmen charakterisierenden Tatbeständen und der Bewertung der Strategien durch das Unternehmen Zusammenhänge nachweisbar sind. Mit anderen Worten: Können Unterschiede zwischen den KMU hinsichtlich der Bewertung der Strategien erklärt werden durch Unterschiede zwischen den KMU hinsichtlich bestimmter ökonomischer Tatbestände? Als solche, ein unterschiedliches Bewertungsverhalten verursachende Tatbestände (im weiteren als Bestimmungsfaktoren bezeichnet) vermuten wir

- den Schwerpunkt der Wirtschaftstätigkeit des Unternehmens, abgebildet in der Zugehörigkeit zu einem bestimmten Wirtschaftszweig bzw. zu einer Branche;

- die Orientierung des Unternehmens auf bestimmte Kundengruppen, bestimmte Vermarktungsformen sowie auf bestimmte Länder im Falle der Entwicklung von Geschäftsbeziehungen mit ausländischen Partnern;

- die Größe des Unternehmens nach Anzahl der Mitarbeiter bzw. dem Jahresumsatz.

Die Untersuchungen wurde in zwei Stufen realisiert. In einer ersten Stufe wurden der Analyse der Zusammenhänge zwischen den Bestimmungsfaktoren und der Bewertung der Strategien die einzelnen Strategien zugrunde gelegt. Die Ergebnisse der entsprechenden Analysen sind in Abschnitt 6.1. zusammengefaßt dargestellt.

Die in der zweiten Stufe realisierte Vorgehensweise verwendet die im Abschnitt 5.2. diskutierte Aggregation der Einzelstrategien zu drei Faktoren (empirische Strategiegruppen). Die Ergebnisse dieser Analyse sind im Abschnitt 6.2. dargelegt.

\subsection{Analyse auf Grundlage der Einzelstrategien}

\subsubsection{Bestimmungsfaktoren Wirtschaftszweig und Strategiebewertung}

Für sieben ausgewählte Wirtschaftszweige (s. Tabelle 12) wurden für die acht bewerteten Strategien die durchschnittlichen Punktbewertungen ermittelt. Für jeden Wirtschaftszweig wurde jene Strategie markiert (= schattierter Hintergrund), welche die höchste Bewertung (= niedrigster Punktwert) erhielt.

Die Strategie 8 (Direkt-Kapitalinvestition zu $100 \%$ ) wird im Durchschnitt der zum jeweiligen Wirtschaftszweig gehörigen KMU in allen Wirtschaftszweigen als eher unwichtig bewertet (größter Durchschnittswert 6, kleinster Durchschnittswert 4,5). Ähnlich das Bild bei Strategie 4 (Franchising: größter Durchschnittswert 5,5 kleinster Durchschnittswert 4,25).

Dagegen wird die Strategie 2 (Export über Handelshäuser und -kommisionäre im Zielland) im Durchschnitt der zum jeweiligen Wirtschaftszweig gehörigen KMU in fünf von sieben Wirtschaftszweigen als am wichtigsten bewertet. Die Durchschnittswerte liegen relativ dicht zusammen (zwischen 2,231 und 3,067). Die Unterschiede in der Bewertung der weiteren Strategien werden in graphischen Darstellung (Abbildung 3) deutlich. 
durchschnittliche Bewertung je Wirtschaftszweig (Anzahl valider Fälle) für Strategie Nr.

\begin{tabular}{|c|l|c|c|c|c|c|c|c|}
\hline Nr. & SIC-Code & 22 & 28 & 29 & 31 & 33 & 72 & 74 \\
\hline 1 & $\begin{array}{l}\text { Export über europäische Handels- } \\
\text { häuser und -kommissionäre }\end{array}$ & $\begin{array}{c}2,875 \\
(8)\end{array}$ & $\begin{array}{c}2,929 \\
(14)\end{array}$ & $\begin{array}{c}3,833 \\
(12)\end{array}$ & $\begin{array}{c}3,857 \\
(14)\end{array}$ & $\begin{array}{c}3,462 \\
(13)\end{array}$ & $\begin{array}{c}4,286 \\
(7)\end{array}$ & $\begin{array}{c}3,739 \\
(46)\end{array}$ \\
\hline 2 & $\begin{array}{l}\text { Export über Handelshäuser und } \\
\text {-kommissionäre im Zielland }\end{array}$ & $\begin{array}{c}2,875 \\
(8)\end{array}$ & $\begin{array}{c}2,750 \\
(16)\end{array}$ & $\begin{array}{c}2,833 \\
(12)\end{array}$ & $\begin{array}{c}2,286 \\
(14)\end{array}$ & $\begin{array}{c}2,231 \\
(13)\end{array}$ & $\begin{array}{c}2,625 \\
(8)\end{array}$ & $\begin{array}{c}3,067 \\
(45)\end{array}$ \\
\hline 3 & $\begin{array}{l}\text { Lizenz- und Technologietransfer- } \\
\text { Verträge mit ausländischen Partnern }\end{array}$ & $\begin{array}{c}3,167 \\
(6)\end{array}$ & $\begin{array}{c}3,273 \\
(11)\end{array}$ & $\begin{array}{c}4,364 \\
(11)\end{array}$ & $\begin{array}{c}3,462 \\
(13)\end{array}$ & $\begin{array}{c}4,000 \\
(12)\end{array}$ & $\begin{array}{c}2,444 \\
(9)\end{array}$ & $\begin{array}{c}3,087 \\
(46)\end{array}$ \\
\hline 4 & Franchising & $\begin{array}{c}4,600 \\
(5)\end{array}$ & $\begin{array}{c}5,364 \\
(11)\end{array}$ & $\begin{array}{c}4,200 \\
(10)\end{array}$ & $\begin{array}{c}5,545 \\
(11)\end{array}$ & $\begin{array}{c}5,077 \\
(13)\end{array}$ & $\begin{array}{c}4,667 \\
(6)\end{array}$ & $\begin{array}{c}4,529 \\
(34)\end{array}$ \\
\hline 5 & $\begin{array}{l}\text { Rahmenverträge über Absatz und } \\
\text { Vertrieb (Kontraktmanagement) }\end{array}$ & $\begin{array}{c}2,375 \\
(8)\end{array}$ & $\begin{array}{c}3,917 \\
(12)\end{array}$ & $\begin{array}{c}2,909 \\
(11)\end{array}$ & $\begin{array}{c}3,385 \\
(13)\end{array}$ & $\begin{array}{c}3,071 \\
(14)\end{array}$ & $\begin{array}{c}2,000 \\
(10)\end{array}$ & $\begin{array}{c}3,372 \\
(43)\end{array}$ \\
\hline 6 & $\begin{array}{l}\text { Eigene Vertriebsorganisation im } \\
\text { Zielland }\end{array}$ & $\begin{array}{c}4,500 \\
(6)\end{array}$ & $\begin{array}{c}3,455 \\
(11)\end{array}$ & $\begin{array}{c}3,833 \\
(12)\end{array}$ & $\begin{array}{c}2,417 \\
(12)\end{array}$ & $\begin{array}{c}3,769 \\
(13)\end{array}$ & $\begin{array}{c}3,286 \\
(7)\end{array}$ & $\begin{array}{c}3,875 \\
(40)\end{array}$ \\
\hline 7 & $\begin{array}{l}\text { Beteiligung an ausländischen } \\
\text { Unternehmen (Joint Venture) }\end{array}$ & $\begin{array}{c}4,625 \\
(8)\end{array}$ & $\begin{array}{c}3,818 \\
(11)\end{array}$ & $\begin{array}{c}4,000 \\
(10)\end{array}$ & $\begin{array}{c}3,769 \\
(13)\end{array}$ & $\begin{array}{c}4,500 \\
(14)\end{array}$ & $\begin{array}{c}4,000 \\
(8)\end{array}$ & $\begin{array}{c}3,340 \\
(47)\end{array}$ \\
\hline 8 & Direkt-Kapitalinvestition zu 100\% & $\begin{array}{c}6,000 \\
(69\end{array}$ & $\begin{array}{c}5,083 \\
(12)\end{array}$ & $\begin{array}{c}4,700 \\
(10)\end{array}$ & $\begin{array}{c}4,833 \\
(12)\end{array}$ & $\begin{array}{c}5,500 \\
(12)\end{array}$ & $\begin{array}{c}5,167 \\
(6)\end{array}$ & $\begin{array}{c}4,526 \\
(38)\end{array}$ \\
\hline
\end{tabular}

Tab. 12: Durchschnittliche Bewertung der Strategien in ausgewählten Wirtschaftszweigen

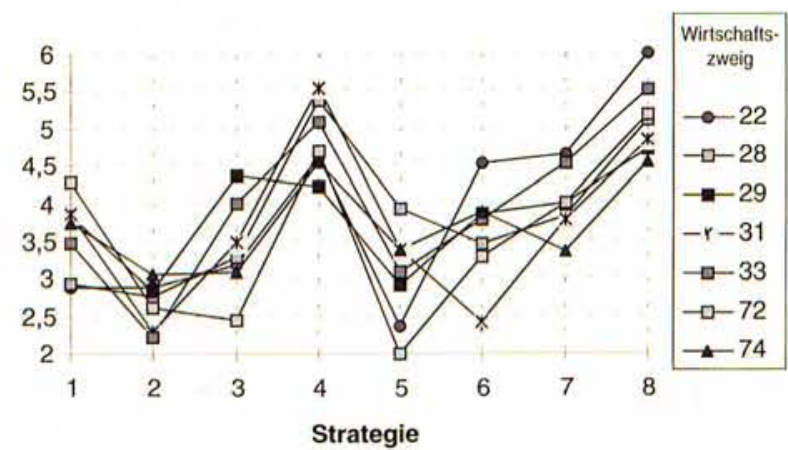

Abb. 3: Strategiebewertungsprofile für ausgewählte Wirtschaftszweige

Deutliche Unterschiede zwischen den Wirtschaftszweigen sind erkennbar bei den Bewertungen der Strategien 3 (Lizenz- und Technologietransfer-Verträge mit ausländischen Partern), 5 (Rahmenverträge über Absatzund Vertrieb; Kontraktmanagement), und Strategie 6 (eigene Vertriebsorganisation im Zielland - die Spannweite der durchschnittlichen Bewertungen beträgt hier ca. 2 Punkte). Die Spannweite der Bewertung der Strategien 1 (Export über europäische Handelshäuser und -kommissionäre) und 7 (Beteiligung an ausländischen Unternehmen; Joint Venture) beträgt im Vergleich der Wirtschaftszweige ca. 1,4 Punkte.

Ob die aus deskriptiver Sicht diagnostizierten Unterschiede zwischen den Wirtschaftszweigen in der Bewertung der Strategien 1, 3, 5, 6 und 7 signifikant sind, läßt sich bei gegebener Datenlage statistisch nicht nachweisen. Im Wirtschaftszweig 74 „Architektur- und Ingenieurbüros" vermuten wir ein - empirisch noch detaillierter zu bestimmendes - Potential für deutsche Direktinvestitionen in asiatischen Ländern. Jeweils drei der befragten Architektur- und Ingenieurbüros gaben in einer weiteren Frage unserer Erhebung an (ohne dies inhaltlich zu präzisieren), daß sie in den ASEAN-Staaten, China oder Japan tätig wären. Im Rahmen der Studie von Borrmann wurden keine Direktinvestitionen dieses Wirtschaftszweiges in Asien bekannt; wir können zunächst nur vermuten, daß diese Investitionen wegen ihres geringen Volumens - sofern vorhanden - von der Statistik der Bundesbank wie auch des Firmeninformationssystems FIS der Industrie- und Handelskammern nicht erfaßt werden (vgl. BORRMANN et al., S. 46 ff).

\subsubsection{Branche und Strategiebewertung}

Für die in Abschnitt 3.2. disjunkt gruppierten Branchen (siehe Tabelle 2) wurden für die acht bewerteten Strategien die durchschnittlichen Punktbewertungen ermittelt (Tabelle 13). Die Strategie mit der geringsten Bewertung (hohe Bedeutung) wurde in jeder Branche durch einen schattierten Hintergrund markiert

\begin{tabular}{|c|c|c|c|c|c|}
\hline \multirow[b]{2}{*}{$\mathrm{Nr}$. } & \multirow[b]{2}{*}{ Strategie } & \multicolumn{4}{|c|}{ Branche } \\
\hline & & 1 & 2 & 3 & 4 \\
\hline 1 & $\begin{array}{l}\text { Export über europäische Handels- } \\
\text { häuser und -kommissionäre }\end{array}$ & $\begin{array}{c}3,627 \\
(58)\end{array}$ & $\begin{array}{c}2,931 \\
(29)\end{array}$ & $\begin{array}{c}4.000 \\
(45)\end{array}$ & $\begin{array}{c}3,333 \\
(6)\end{array}$ \\
\hline 2 & $\begin{array}{l}\text { Export über Handelshäuser und } \\
\text {-kommissionäre im Zielland }\end{array}$ & $\begin{array}{c}2,263 \\
(57)\end{array}$ & $\begin{array}{c}2,138 \\
(29)\end{array}$ & $\begin{array}{c}3,341 \\
(44)\end{array}$ & $\begin{array}{c}2,778 \\
(9)\end{array}$ \\
\hline 3 & $\begin{array}{l}\text { Lizenz- und Technologietransfer- } \\
\text { Verträge mit ausländischen Partnern }\end{array}$ & $\begin{array}{c}3.727 \\
(55)\end{array}$ & $\begin{array}{c}4,222 \\
(27)\end{array}$ & $\begin{array}{c}2.739 \\
(46)\end{array}$ & $\begin{array}{c}2,375 \\
(8)\end{array}$ \\
\hline 4 & Franchising & $\begin{array}{c}5.133 \\
(45)\end{array}$ & $\begin{array}{c}5,080 \\
(25)\end{array}$ & $\begin{array}{c}4,694 \\
(36)\end{array}$ & $\begin{array}{c}3,833 \\
(6)\end{array}$ \\
\hline 5 & $\begin{array}{l}\text { Rahmenverträge über Absatz und } \\
\text { Vertrieb (Kontraktmanagement) }\end{array}$ & $\begin{array}{c}3.482 \\
(54)\end{array}$ & $\begin{array}{c}2,567 \\
(30)\end{array}$ & $\begin{array}{c}3,217 \\
(46)\end{array}$ & $\begin{array}{c}2,167 \\
(6)\end{array}$ \\
\hline 6 & $\begin{array}{l}\text { Eigene Vertriebsorganisation im } \\
\text { Zielland }\end{array}$ & $\begin{array}{c}3,286 \\
(49)\end{array}$ & $\begin{array}{c}4,107 \\
(28)\end{array}$ & $\begin{array}{c}4,256 \\
(43)\end{array}$ & $\begin{array}{c}3,000 \\
(8)\end{array}$ \\
\hline 7 & $\begin{array}{l}\text { Beteiligung an ausländischen } \\
\text { Unternehmen (Joint Venture) }\end{array}$ & $\begin{array}{c}3,539 \\
(52)\end{array}$ & $\begin{array}{c}4,462 \\
(26)\end{array}$ & $\begin{array}{c}3,628 \\
(51)\end{array}$ & $\begin{array}{c}3,250 \\
(8)\end{array}$ \\
\hline 8 & Direkt-Kapitalinvestition zu 100 \& & $\begin{array}{c}4,854 \\
(48)\end{array}$ & $\begin{array}{c}5,040 \\
(25)\end{array}$ & $\begin{array}{c}5,073 \\
(41)\end{array}$ & $\begin{array}{c}4,126 \\
(8)\end{array}$ \\
\hline
\end{tabular}

Tab. 13: Durchschnittliche Bewertung der Strategien in den disjunkt gruppierten Branchen 
Hierbei werden deutlichere Unterschiede in der Bewertung zwischen den definierten Branchen erkennbar. Die Spannweite der durchschnittlichen Bewertungen beträgt jeweils ca. 1 bis 2 Punkte (vgl. Abbildung 4).
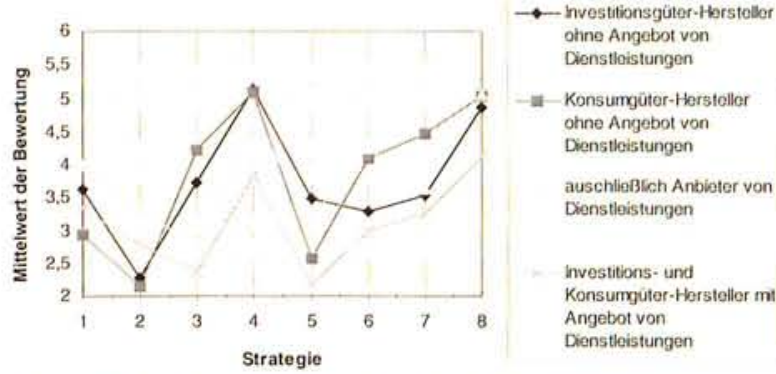

Abb. 4: Strategiebewertungsprofile für disjunkt gruppierte Branchen

Um herauszufinden, ob die zu den einzelnen Branchen gehörenden KMU bestimmte Strategien signifikant unterschiedlich bewerten, wurden die entsprechenden Chi-Quadrat-Unabhängigkeitstests durchgeführt. Ausgeschlossen aus der Analyse wurden wegen der zu geringen Fallzahl die Unternehmen der gemischten Branche „Hersteller von Investitions- und Konsumgütern sowie Anbieter von Dienstleistungen" (Branche 4). Weiterhin wurde der Analyse eine aggregierte Strategiebewertungsskala (3-er Skala) zugrunde gelegt, in welcher noch einmal die Bewertungen 1 und 2, 3 und 4 sowie 5 und 6 zusammengefaßt wurden. Die wichtigsten Maßzahlen aus den Tests sind in Tabelle 14 dargestellt.

\begin{tabular}{|c|c|c|c|c|c|}
\hline & \multicolumn{3}{|c|}{ Maße für Zusammenhangsanalyse } & \multicolumn{2}{|c|}{$\begin{array}{c}\text { Einhaltung } \\
\text { Testvoraussetzung }\end{array}$} \\
\hline $\begin{array}{l}\text { Stra- } \\
\text { tegie- } \\
\mathrm{Nr} \text {. }\end{array}$ & $\begin{array}{l}\text { Chi-Qua- } \\
\text { drat nach } \\
\text { Pearson }\end{array}$ & $\begin{array}{c}\text { Cramer's } \\
V\end{array}$ & $\begin{array}{l}\text { beobachtetes } \\
\text { Signifikanz- } \\
\text { niveau fuir } \\
\text { Chi-Quadrat }\end{array}$ & $\begin{array}{l}\text { Min. } \\
\text { erwart. } \\
\text { Häufig- } \\
\text { keit }\end{array}$ & $\begin{array}{l}\text { Anteil mit } \\
\text { erwarteter } \\
\text { Häuf. }<5\end{array}$ \\
\hline 1 & 4,608 & 0.132 & 0,329 & 6,59 & \\
\hline 2 & 10,023 & 0.196 & 0.040 & 5,35 & \\
\hline 3 & 14,129 & 0.235 & 0,007 & 7,38 & \\
\hline 4 & 5,359 & 0.159 & 0.252 & 1,65 & $\begin{array}{l}3 \text { von } 9 \\
(33,3 \%)\end{array}$ \\
\hline 5 & 5,205 & 0.141 & 0,267 & 7.62 & \\
\hline 6 & 7,111 & 0,172 & 0,130 & 6,30 & \\
\hline 7 & 4.865 & 0.137 & 0,301 & 7,66 & \\
\hline 8 & 1,783 & 0,088 & 0.776 & 2.19 & $\begin{array}{l}4 \text { von } 9 \\
(44,4 \%)\end{array}$ \\
\hline
\end{tabular}

Tab. 14: Ausgewählte Maßzahlen der Chi-Quadrat-Unabhängigkeitstests für Branche mit Bewertung der Strategien 1 bis 8

Signifikante Unterschiede zwischen den Branchen hinsichtlich der Bewertung der Strategien lassen sich nur für die Strategien 2 (Export über Handelshäuser und -kommisionäre im Zielland) und 3 (Lizenz- und Technologietransferverträge mit ausländichen Partnern) nachweisen. Für KMU der Branchen 1 (Hersteller von Investitionsgütern) und 2 (Hersteller von Investitionsgütern) ist die Strategie 2 (Export über Handelshäuser und -kommisionäre im Zielland) signifikant wichtiger als für reine Dienstleistungsanbieter. 64,9 Prozent der Unternehmen der Branche 1 und 72,4 Prozent der Unternehmen der Branche 2 bewerten die Strategie 2 mit 1 oder 2, wohingegen nur 40,9 Prozent der reinen Dienstlei- stungsanbieter diese Strategie mit 1 oder 2 bewerten. Andererseits ist fur die reinen Dienstleistungsanbieter (Branche 3) die Strategie 3 (Lizenz- und Technologietransferverträge mit ausländichen Partnern) signifikant wichtiger als für die produzierenden KMU der Branchen 1 und 2. 52,2 Prozent der reinen Dienstleistungsanbieter bewerten die Strategie 3 mit 1 oder 2. In den Branchen 1 und 2 betragen die entsprechenden Anteile nur 30,9 bzw. 33,3 Prozent.

Die in Tabelle 14 dargestellten Ergebnisse lassen weitere Zusammenhänge vermuten, deren Signifikanz aber auf Grund der geringen Fallzahlen statistisch nicht nachgewiesen werden kann. Konsumgüter- und Investitionsgüterhersteller (mit und ohne Angebot von Dienstleistungen) unterscheiden sich hinsichtlich der Bewertung der Strategien 3 (Lizenz- und Technologietransferverträge mit ausländischen Partnern) und 5 (Kontraktmanagement).

\subsubsection{Bestimmungsfaktoren Kunden, Vermarktungsformen und Länderwahl}

Analysen in Form des Mittelwertvergleichs und des ChiQuadrat-Testes wie in den Abschnitten 6.1.1 und 6.1.2 zeigten keine Abhängigkeiten der Strategiewahl von den Kundengruppen der Unternehmen oder den Vermarktungsformen der KMU (Definitionen s. Abschnitt 3.3.) - stets zeigte sich wieder die bereits in Abschnitt 5.1. beschriebene Rangfolge der Strategien.

Auch die Marktbedingungen des jeweiligen Ziellandes könnten einen Einfluß auf die Strategie zur Erschließung des Potentials ausländischer Märkte haben - eine Hypothese, die nach den Ergebnissen unserer Auswertungen nicht verifiziert werden konnte. Die Bewertung der acht Strategien erfolgte - unabhängig von der Länderwahl der befragten $\mathrm{KMU}$ - im wesentlichen wiederum in der bereits genannten Rangfolge.

Die Autoren verzichten an dieser Stelle auf die Dokumentation jener Analysen, welche letztlich keine statistisch gesicherten Ergebnisse brachten.

\subsubsection{Bestimmungsfaktoren Mitarbeiterzahl und Jahresumsatz} Unterschiede in der Bewertung der Strategien werden wiederum sichtbar durch eine Klassifizierung der Unternehmen nach ihrer Mitarbeiterzahl, deren Ergebnisse in Tabelle 15 und Abbildung 5 dargestellt werden.

\begin{tabular}{|c|c|c|c|c|c|c|}
\hline \multirow[b]{2}{*}{$\mathrm{Nr}$. } & \multirow[b]{2}{*}{ Strategie } & \multicolumn{5}{|c|}{ Mitarbeiterzahl } \\
\hline & & $10 \cdot 49$ & $50-99$ & $100-249$ & $250 \cdot 499$ & $500-999$ \\
\hline 1 & $\begin{array}{l}\text { Export über europäische } \\
\text { Handelshäuser }\end{array}$ & $\begin{array}{c}3,366 \\
(82)\end{array}$ & $\begin{array}{c}3,500 \\
(22)\end{array}$ & $\begin{array}{c}3.759 \\
(29)\end{array}$ & $\begin{array}{c}4,400 \\
(10)\end{array}$ & $\begin{array}{c}4.875 \\
(8)\end{array}$ \\
\hline 2 & $\begin{array}{l}\text { Export über Handelshäuser } \\
\text { im Zielland }\end{array}$ & $\begin{array}{c}2.786 \\
(84)\end{array}$ & $\begin{array}{l}2,64 \\
(25)\end{array}$ & $\begin{array}{c}2,143 \\
(28)\end{array}$ & $\begin{array}{c}3.125 \\
(8)\end{array}$ & $\begin{array}{c}3,125 \\
(8)\end{array}$ \\
\hline 3 & $\begin{array}{l}\text { Lizenz- und Technologie- } \\
\text { transfer-Verträge }\end{array}$ & $\begin{array}{c}3,247 \\
(77)\end{array}$ & $\begin{array}{c}3,808 \\
(26)\end{array}$ & $\begin{array}{c}3.320 \\
(25)\end{array}$ & $\begin{array}{l}3.556 \\
(9)\end{array}$ & $\begin{array}{c}3,333 \\
(9)\end{array}$ \\
\hline 4 & Franchising & $\begin{array}{c}4.672 \\
(64)\end{array}$ & $\begin{array}{l}5,000 \\
(22)\end{array}$ & $\begin{array}{c}4,909 \\
(22)\end{array}$ & $\begin{array}{c}6,000 \\
(5)\end{array}$ & $\begin{array}{c}5,000 \\
(8)\end{array}$ \\
\hline 5 & $\begin{array}{l}\text { Rahmenverträge über Absatz u. } \\
\text { Vertrieb (Kontraktmanagement) }\end{array}$ & $\begin{array}{c}2,987 \\
(79)\end{array}$ & $\begin{array}{c}3,077 \\
(26)\end{array}$ & $\begin{array}{l}3,120 \\
(25)\end{array}$ & $\begin{array}{c}3,750 \\
(8)\end{array}$ & $\begin{array}{l}3.556 \\
(9)\end{array}$ \\
\hline 6 & $\begin{array}{l}\text { Eigene Vertriebsorganisation } \\
\text { im Zielland }\end{array}$ & $\begin{array}{c}3,986 \\
(72)\end{array}$ & $\begin{array}{l}3,917 \\
(24)\end{array}$ & $\begin{array}{c}3.000 \\
(25)\end{array}$ & $\begin{array}{c}3.444 \\
(9)\end{array}$ & $\begin{array}{l}2,333 \\
\text { (9) }\end{array}$ \\
\hline 7 & $\begin{array}{l}\text { Beteiligung an ausländischen } \\
\text { Unternehmen (Joint Venture) }\end{array}$ & $\begin{array}{c}3,988 \\
(80)\end{array}$ & $\begin{array}{c}3,500 \\
(26)\end{array}$ & $\begin{array}{c}3.542 \\
(24)\end{array}$ & $\begin{array}{c}3,333 \\
\text { (9) }\end{array}$ & $\begin{array}{c}3,250 \\
(8)\end{array}$ \\
\hline 8 & $\begin{array}{l}\text { Direkt-Kapitalinvestition } \\
\text { (zu } 100 \text { \%) }\end{array}$ & $\begin{array}{l}5,211 \\
(71)\end{array}$ & $\begin{array}{l}4,636 \\
(22)\end{array}$ & $\begin{array}{c}4,591 \\
(22)\end{array}$ & $\begin{array}{c}5,375 \\
(8)\end{array}$ & $\begin{array}{c}3,250 \\
(8)\end{array}$ \\
\hline
\end{tabular}

Tab. 15: Durchschnittliche Bewertung der Strategien in fünf Unternehmensgrößen-Klassen, gruppiert nach Mitarbeiterzahl 
Die großen brandenburgischen und Berliner Unternehmen (500 und mehr Mitarbeiter) bewerten entgegen den Unternehmen mit weniger als 500 Mitarbeitern die kapitalintensiveren Formen einer Strategie der Erschließung ausländischer, in unserer Erhebung asiatischer, Märkte für ihr Unternehmen eher als wichtig.

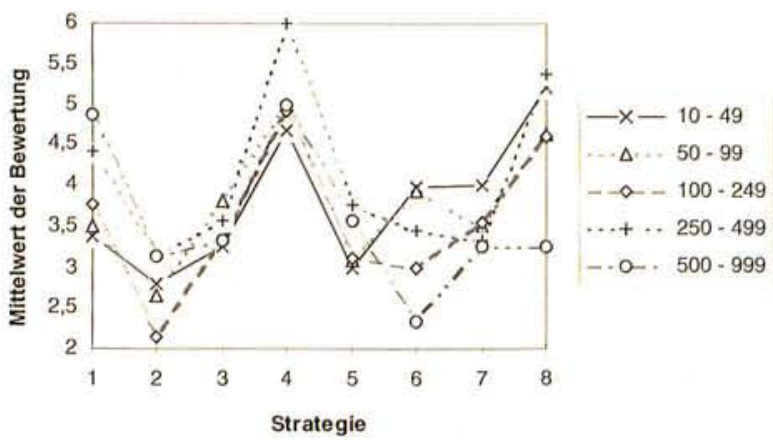

Abb. 5: Strategie-Bewertungsprofil für Unternehmensgrößenklassen (nach Mitarbeiterzahl)

Ein ähnliches Profil (Abbildung 6) zur Bewertung der acht Strategien erhalten wir, wenn wir die befragten Unternehmen nach dem Umsatz des Jahres 1995 klassifizieren (dazu Tabelle 16).

\begin{tabular}{|c|c|c|c|c|c|c|}
\hline \multirow[b]{2}{*}{$\mathrm{Nr}$. } & \multirow[b]{2}{*}{ Strategie } & \multicolumn{5}{|c|}{ Jahresumsatz 1995 in DM (valide Fälle) } \\
\hline & & $<1$ Mio. & $\begin{array}{c}1 \text { bis } \\
<5 \text { Mio }\end{array}$ & $\begin{array}{r}5 \text { bis } \\
<10 \text { Mio } \\
\end{array}$ & $\begin{array}{c}10 \text { bis } \\
<50 \text { Mio }\end{array}$ & $\begin{array}{c}>= \\
50 \text { Mio }\end{array}$ \\
\hline 1 & $\begin{array}{l}\text { Export über europäische } \\
\text { Handelshäuser }\end{array}$ & & & $\begin{array}{c}3,364 \\
(22) \\
\end{array}$ & $\begin{array}{c}3,244 \\
(41)\end{array}$ & $\begin{array}{c}4,810 \\
(21)\end{array}$ \\
\hline 2 & $\begin{array}{l}\text { Export über Handelshäuser } \\
\text { im Zielland }\end{array}$ & $\begin{array}{c}2,182 \\
(11)\end{array}$ & $\begin{array}{c}3,306 \\
(55)\end{array}$ & $\begin{array}{c}2,208 \\
(24)\end{array}$ & $\begin{array}{c}2,250 \\
(40)\end{array}$ & $\begin{array}{c}3,000 \\
(20)\end{array}$ \\
\hline 3 & $\begin{array}{l}\text { Lizenz- und Technologie- } \\
\text { transfer-Verträge }\end{array}$ & $\begin{array}{c}3,091 \\
(11)\end{array}$ & $\begin{array}{c}3,122 \\
(49)\end{array}$ & $\begin{array}{c}4,080 \\
(25)\end{array}$ & $\begin{array}{c}3,432 \\
(37)\end{array}$ & $\begin{array}{c}3.190 \\
(21)\end{array}$ \\
\hline 4 & Franchising & $\begin{array}{c}4,900 \\
(10)\end{array}$ & $\begin{array}{c}4,581 \\
(43)\end{array}$ & $\begin{array}{c}5,111 \\
(18)\end{array}$ & $\begin{array}{c}5,129 \\
(31)\end{array}$ & $\begin{array}{l}4,875 \\
(16)\end{array}$ \\
\hline 5 & $\begin{array}{l}\text { Rahmenverträge ïber Absatz u. } \\
\text { Vertrieb (Kontraktmanagement) }\end{array}$ & $\begin{array}{c}2,583 \\
(12)\end{array}$ & $\begin{array}{c}3,100 \\
(50)\end{array}$ & $\begin{array}{c}2,783 \\
(23)\end{array}$ & $\begin{array}{c}3,385 \\
(39)\end{array}$ & $\begin{array}{l}3,300 \\
(20)\end{array}$ \\
\hline 6 & $\begin{array}{l}\text { Eigene Vertriebsorganisation } \\
\text { im Zielland }\end{array}$ & $\begin{array}{l}4,182 \\
\text { (11) }\end{array}$ & $\begin{array}{c}4,205 \\
(44)\end{array}$ & $\begin{array}{c}3,913 \\
(23)\end{array}$ & $\begin{array}{c}3,405 \\
(37)\end{array}$ & $\begin{array}{l}2,571 \\
(21)\end{array}$ \\
\hline 7 & $\begin{array}{l}\text { Beteiligung an ausländischen } \\
\text { Unternehmen (Joint Venture) }\end{array}$ & $\begin{array}{c}3,385 \\
(13)\end{array}$ & $\begin{array}{c}4,040 \\
(50)\end{array}$ & $\begin{array}{c}3,750 \\
(24)\end{array}$ & $\begin{array}{c}3,865 \\
(37)\end{array}$ & $\begin{array}{l}2,950 \\
(20)\end{array}$ \\
\hline 8 & $\begin{array}{l}\text { Direkt-Kapitalinvestition } \\
\text { (zu } 100 \%)\end{array}$ & $\begin{array}{c}5,600 \\
(10)\end{array}$ & $\begin{array}{c}5.087 \\
(46)\end{array}$ & $\begin{array}{c}5.450 \\
(20)\end{array}$ & $\begin{array}{c}4,618 \\
(34)\end{array}$ & $\begin{array}{c}4,333 \\
(18)\end{array}$ \\
\hline
\end{tabular}

Tab. 16: Durchschnittliche Bewertung der Strategien in fünf Klassen nach Jahresumsatz 1995

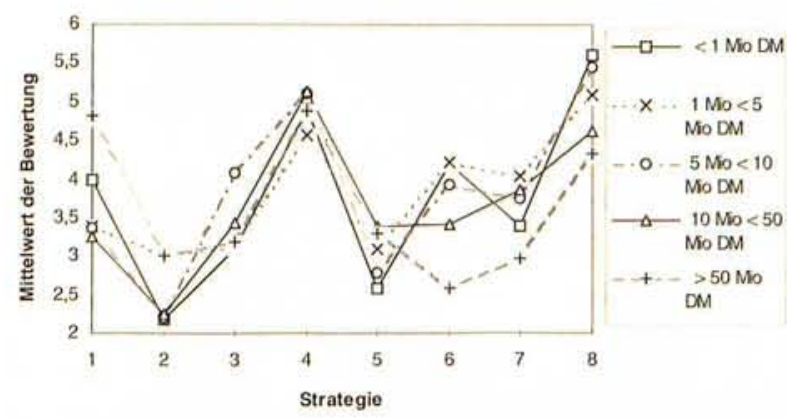

Abb. 6: Strategie-Bewertungsprofil für Unternehmensgrößenklassen (nach Jahresumatz 1995)

\subsection{Analysen auf Grundlage der empirisch ermittelten Strategiegruppen}

6.2.1 Gruppierung der Unternehmen nach den empirisch ermittelten Strategiegruppen

Die im Abschnitt 5.2. mittels der Faktorenanalyse empirisch separierten drei Strategiegruppen

- Strategie-Gruppe 1 (Faktor 1): Dominanz kapitalintensiver Strategien mit unterschiedlichen Kontrollrechten,

- Strategie-Gruppe 2 (Faktor 2): Dominanz der Strategien mit geringer Kapitalintensität und geringen Kontrollrechten und

- Strategie-Gruppe 3 (Faktor 3): Dominanz der Strategien mit geringer Kapitalintensität und unterschiedlichen Kontrollrechten

werden der folgenden Gruppierung der befragten Unternehmen zugrunde gelegt. Nach Ausgliederung der Missing-Fälle in der Faktorenanalyse konnten nur für 111 Unternehmen die Faktorwerte für alle drei Faktoren ermittelt werden. Jedes dieser Unternehmen wird jetzt bezüglich der Bewertung der Strategien nicht mehr durch die für die einzelnen Strategien vergebenen Punktwerte charakterisiert, sondern durch die für jeden Faktor berechneten Werte (Faktorwerte). Ein Faktorwert von Null bedeutet ein durchschnittliches (im Durchschnitt aller analysierten Unternehmen) Bewertungsverhalten hinsichtlich der durch den betreffenden Faktor dargestellten Strategiegruppe. Ein Faktorwert von größer als Null bedeutet, unter Berücksichtigung der Konstruktion der Skala (vgl. Abschnitt 5), eine uiberdurchschnittlich geringe Bewertung der entsprechenden Strategiegruppe. Umgekehrt bedeutet ein Faktorwert von kleiner als Null eine überdurchschnittlich hohe Bewertung der entsprechenden Strategiegruppe.

Die Gruppierung der 111 Unternehmen unter Anwendung des Verfahrens der Clusteranalyse wurde in zwei Stufen realisiert. In der ersten Stufe wurden alle 111 Unternehmen einer hierarchischen Clusterung unter Anwendung des Ward-Algorithmus unterzogen. Nach Auswertung des entsprechenden Dendrogramms wurde die Anzahl der Cluster mit vier bestimmt. In der zweiten Stufe wurde die Zusammensetzung der vier Cluster unter Anwendung des k-Mean-Algorithmus bestimmt.

Im Ergebnis der Clusteranalyse ergibt sich eine Aufteilung der 111 Unternehmen in vier, hinsichtlich der Strategiebewertung unterschiedlich konfigurierte Cluster (siehe Tabelle 17).

\begin{tabular}{|crrcc|}
\hline Cluster & Faktor 1 & Faktor 2 & Faktor 3 & Anzahl \\
\hline 1 &, 74 & 1,37 &, 26 & 20 \\
2 &, 53 &,- 82 &, 68 & 34 \\
3 &, 03 &,- 12 & $-1,33$ & 29 \\
4 & $-1,21$ &, 14 &, 37 & 28 \\
\hline
\end{tabular}

Tab. 17: Mittelwertvektoren der Cluster und Anzahl der Unternehmen im Cluster

Cluster 1: Die im Cluster 1 zusammengefaßten Unternehmen bewerten vor allem die nicht-kapitalintensiven Strategien des Exports ïber Handelshäuser (Faktor 2) 
überdurchschnittlich gering. Ebenfalls überdurchschnittlich gering, aber nicht so ausgeprägt wie bei Faktor 2, werden die kapitalintensiven Strategien (Faktor 1) sowie die sonstigen nicht-kapitalintensiven Strategien (Faktor 3) bewertet.

Cluster 2: Die in diesem Cluster zusammengefaßten Unternehmen bewerten überdurchschnittlich hoch die nicht-kapitalintensiven Strategien des Exports über Handelshäuser (Faktor 2), wohingegen die kapitalintensiven Strategien (Faktor 1) sowie die sonstigen nicht-kapitalintensiven Strategien (Faktor 3) iiberdurchschnittlich gering bewertet werden.

Cluster 3: Unternehmen dieses Clusters bewerten überdurchschnittlich hoch die sonstigen nicht-kapitalintensiven Strategien (Faktor 3). Etwa durchschnittlich bewertet werden die kapitalintensiven Strategien (Faktor 1) sowie die nicht-kapitalintensiven Strategien des Exports über Handelshäuser (Faktor 2).

Cluster 4: Eine deutlich ausgeprägte überdurchschnittlich hohe Bewertung der kapitalintensiven Strategien (Faktor 1) kennzeichnet die Unternehmen des Clusters 4. Die nicht-kapitalintensiven Strategien (Faktor 2) werden etwa durchschnittlich bewertet, während die sonstigen nicht-kapitalintensiven Strategien (Faktor 3) überdurchschnittlich gering bewertet werden.

\subsubsection{Bestimmungsfaktoren für die Clusterzugehörigkeit}

Nach der Separierung von vier, hinsichtlich der Strategiebewertung unterschiedlich konfigurierten Gruppen (Cluster) von Berliner und brandenburgischen KMU interessiert die Frage nach den Gründen für die Zugehörigkeit der einzelnen Unternehmen zu einem bestimmten Cluster: Mit anderen Worten: Können die Unterschiede in der Zuordnung der einzelnen Unternehmen zu den Clustern durch Unterschiede zwischen den KMU hinsichtlich bestimmter ökonomischer Tatbestände erklärt werden? Als solche, eine unterschiedliche Zuordnung erklärenden Tatbestände (im weiteren als Bestimmungsfaktoren für die Clusterzugehörigkeit bezeichnet) nahmen wir die in Tabelle 18 dargestellten ökonomischen Merkmale der befragten KMU (vgl. oben, Abschnitt 2) an.

Im Ergebnis einer CHAID-Analyse (Chi-squared Automatic Interaction Detector) konnte in der ersten Ebene für drei der acht vermuteteten Bestimmungsfaktoren ein signifikanter Zusammenhang (beobachtetes Signifikanzniveau für Chi-square kleiner 0,05; siehe auch Tabelle 19) mit der Zugehörigkeit zu den Clustern diagnostiziert werden. Am deutlichsten nachweisbar ist insbesondere der Zusammenhang zwischen der Clusterzugehörigkeit und der Zuordnung zu einer der zwei Branchengruppen Investitionsgüter- bzw. Konsumgüterhersteller

\begin{tabular}{|c|c|c|}
\hline Bestimmungsfaktor & Ausprägungen des Bestimmungsfaktors & $\begin{array}{l}\text { Codierung des } \\
\text { Bestimmungsfaktors }\end{array}$ \\
\hline Branche & $\begin{array}{l}\text { - reine Investitionsgüter-Hersteller } \\
\text { - reine Konsumgüter-Hersteller } \\
\text { - reine Dienstleistungsanbieter } \\
\text { - Mischtypen (Anbieter von Dienstleistungen } \\
\text { und Hersteller von Invest.- und Konsumgüter) }\end{array}$ & branche 1 \\
\hline $\begin{array}{l}\text { Unternehmensgröße nach } \\
\text { Mitarbeiterzahl }\end{array}$ & $\begin{array}{l}\text { - } 10 \text { bis } 49 \text { Personen } \\
\text { - } 50 \text { bis } 99 \text { Personen } \\
\text { - } 100 \text { bis } 249 \text { Personen } \\
\text { - } 250 \text { bis } 499 \text { Personen } \\
\text { - über } 500 \text { Personen }\end{array}$ & f18_6a \\
\hline $\begin{array}{l}\text { Unternehmensgröße nach } \\
\text { Jahresumsatz }\end{array}$ & $\begin{array}{l}\text { - unter } 1 \text { Mio DM } \\
\text { - } 1 \text { bis unter } 5 \text { Mio DM } \\
\text { - } 5 \text { bis unter } 10 \text { Mio DM } \\
\text { - } 10 \text { bis unter } 50 \text { Mio DM } \\
\text { - über } 50 \text { Mio DM }\end{array}$ & f18_7a \\
\hline Vermarktungsformen & $\begin{array}{l}\text { - nur direkte Vermarktung } \\
\text { - nur über Groß- und Einzelhandel } \\
\text { - Mischtyp (direkte Vermarktung und über } \\
\text { Groß- und Einzelhandel) }\end{array}$ & f18_4 \\
\hline Kundentyp & $\begin{array}{l}\text { - private Endverbraucher (auschließlich bzw. } \\
\text { unter anderem) } \\
\text { - keine privaten Endverbraucher }\end{array}$ & kunden_2 \\
\hline $\begin{array}{l}\text { Exportanteil (wieviel Prozent des } \\
\text { Jahresumsatzes entfallen auf Export?) }\end{array}$ & $\begin{array}{l}-0 \% \\
\text { - größer } 0 \text { bis } 10 \% \\
\text { - größer } 10 \text { bis } 50 \% \\
\text { - über } 50 \%\end{array}$ & f18_7b_k \\
\hline $\begin{array}{l}\text { Ist das Unternehmen national } \\
\text { und/oder international tätig? }\end{array}$ & $\begin{array}{l}\text { - national und international tätig } \\
\text { - nur national tätig }\end{array}$ & filter $2 g$ \\
\hline Alter des Unternehmens & $\begin{array}{l}\text { - jünger als } 9 \text { Jahre } \\
-9 \text { bis unter } 59 \text { Jahre } \\
\text { - } 59 \text { Jahre und älter }\end{array}$ & alter_k \\
\hline
\end{tabular}

Tab. 18: Vermutete Bestimmungsfaktoren für die Clusterzugehörigkeit 
auf der einen Seite und den reinen Dienstleistungsanbietern bzw. Mischtypen auf der anderen Seite (beobachtetes Signifikanzniveau von 0,00015 ).

\begin{tabular}{|c|c|c|}
\hline $\begin{array}{l}\text { Bestimmungs- } \\
\text { faktoren }\end{array}$ & Ausprägungen ${ }^{\text {b) }}$ & $\begin{array}{l}\text { beobachtetes } \\
\text { Signifikanz- } \\
\text { niveau fiir } \\
\text { Chi-Quadrat }{ }^{\mathrm{c})}\end{array}$ \\
\hline Branche & $\begin{array}{l}\text { - Investitions- bzw. Konsumgüter- } \\
\text { hersteller (Codierung in Abb. } 7: 12 \text { ) } \\
\text { - reine Dienstleistungsanbieter und } \\
\text { Mischtypen (Codierung in Abb. } 7: 3-\text {.) }\end{array}$ & 0.00015 \\
\hline $\begin{array}{l}\text { international/ } \\
\text { national tätig } \\
\text { Exportanteil }\end{array}$ & $\begin{array}{l}\text { - national und international tätig } \\
\text { - nur national tätig } \\
-0 \% \text { bis unter } 10 \% \\
\text { - über } 10 \%\end{array}$ & $\begin{array}{l}0,0025 \\
0,0125\end{array}$ \\
\hline $\begin{array}{l}\text { Unternehmens- } \\
\text { alter }\end{array}$ & $\begin{array}{l}\text { - jünger als } 59 \text { Jahre } \\
\text { - } 59 \text { Jahre und älter }\end{array}$ & 0,083 \\
\hline $\begin{array}{l}\text { Unternehmens- } \\
\text { größe nach } \\
\text { Umsatz }\end{array}$ & $\begin{array}{l}\text { - bis unter } 5 \text { Mio. DM } \\
\text { - über } 5 \text { Mio. DM }\end{array}$ & 0.093 \\
\hline
\end{tabular}

Tab. 19: Signifikante Bestimmungsfaktoren für die Clusterzugehörigkeit in der ersten Erklärungsebene a)

a) Die Testvoraussetzung für den Chi-Quadrat-Unabhängigkeitstest ist in allen Tests mit einer kleinsten theoretischen Zellenhäufigkeit von größer 5 erfüllt. b) Bei einem beobachteten Signifikanzniveau für Chi-square von kleiner als 0,10 aggregiert.

c) Die zusätzlich mit der Likelihood-Statistik durchgeführten Tests ergaben nur geringfügige Unterschiede im empirischen Signifikanzniveau gegenüber den Tests mit dem Chi-Quadrat nach Pearson.

Für zwei weitere Bestimmungsfaktoren (Alter des Unternehmens; Unternehmensgröße nach Umsatz) kann in der ersten Ebene noch ein signifikanter Zusammenhang mit der Clusterzugehörigkeit diagnostiziert werden, wenn man ein Signifikanzniveau von 0,10 zugrundelegt. Um in einer zweiten Erklärungsebene weitere signifikante Bestimmungsfaktoren nachzuweisen, sind die Fallzahlen zu gering. Wir haben trotz der Nichterfüllung der Testvoraussetzungen in Abbildung 7 die zweite Erklärungsebene aufgenommen, um auf damit

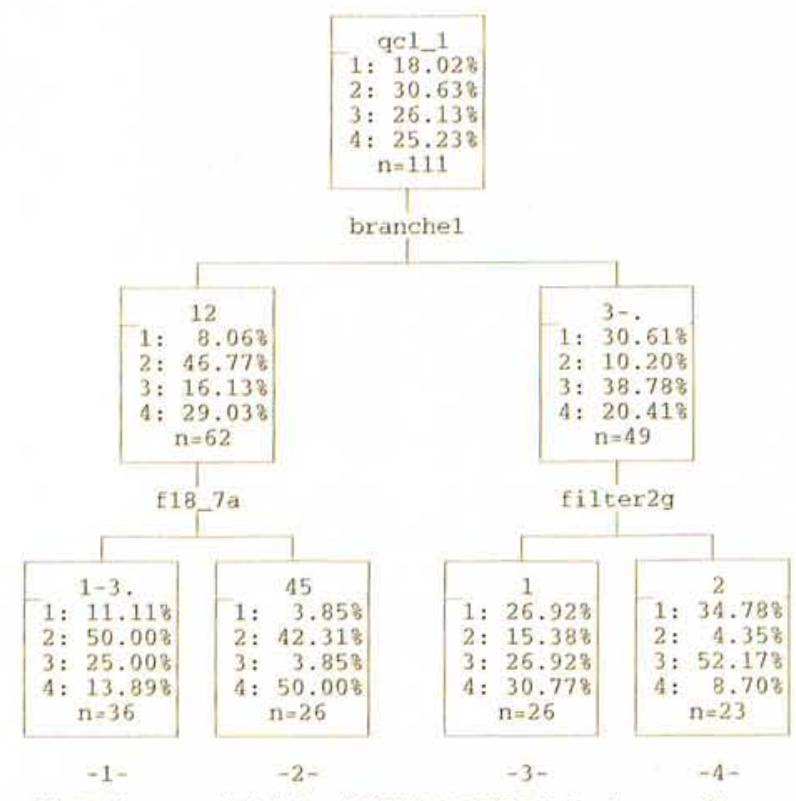

Abb. 7: Clusterzugehörigkeit und CHAID-ANALYSE der Bestimmungsfaktoren.

$q \mathrm{cl} \_1$ bezeichnet die Codierungsvariable für die Clusterzugehörigkeit; die Ziffern I bis 4 (mit Doppelpunkt) bezeichnen die Nummer des Clusters, vgl. oben, Tabelle 17. einen Ausblick auf weitere Untersuchungen, größere Stichproben vorausgesetzt, zu geben.

Für die Gruppe der Unternehmen, die ausschließlich Investitions- bzw. Konsumgüter herstellen, ergibt sich als einziger signifikanter Bestimmungsfaktor (die Verletzung der Testvoraussetzungen außer acht gelassen) die Unternehmensgröße nach dem Jahresumsatz in der Gruppierung bis unter 10 Mio. DM (Codierung in Abbildung 7: 1-3) und 10 Mio. DM und mehr (Codierung in Abbildung 7: 45). Diese 26 Unternehmen mit mehr als 10 Mio. DM Jahresumsatz finden wir fast alle entweder in Cluster 2 (überdurchschnittliche Bewertung der Strategien des Exports über Handelshäuser) oder in Cluster 4 (überdurchschnittliche Bewertung der kapitalintensiven Strategien; vgl. Abschnitt 6.2.1.).

Für die Gruppe der Unternehmen, die ausschließlich Dienstleistungen anbieten bzw. Dienstleistungen anbieten und Investitions- bzw. Konsumgüter herstellen, ergibt sich als einziger relevanter Bestimmungsfaktor die Unterscheidung zwischen Unternehmen, die nur national tätig sind (Codierung in Abbildung 7:2) und solchen Unternehmen, die national und international tätig sind (Codierung in Abbildung 7: 1). Die 23 nur national tätigen Unternehmen finden wir fast alle im Cluster 1 (unterdurchschnittliche Bewertung der Strategien des Exports über Handelshäuser) oder im Cluster 3 (ïberdurchschnittliche Bewertung der sonstigen nichtkapitalintensiven Strategien). Als Hypothese für weitere empirische Analysen größerer Samples leiten wir aus der Abbildung 7 folgende Aussage ab: Nur national tätige Anbieter von Dienstleistungen präferieren für die Erschließung internationaler (asiatischer) Märkte insbesondere verschiedene nicht-kapitalintensive Strategien (vgl. Tabelle 11: Lizenz- und Technologietransferverträge, Kontraktmanagement und evtl. Franchising).

In den Untersuchungen anderer Autoren konnte der bestimmende Einfluß der Unternehmensvariablen Jahresumsatz und Mitarbeiterzahl auf die Strategiewahl bereits mehrfach nachgewiesen werden. Die den Autoren bekannten Untersuchungen im deutschen Sprachraum fanden fast alle nur innerhalb weniger Wirtschaftszweige statt (vgl. BAMBERGER u. EVERS 1994, BEUTEL. 1988, BORRMANN et al. 1996, JENNER 1994, SAUER 1991). Dagegen beteiligten sich an unserer schriftlichen Befragung 269 Unternehmen aus insgesamt 18 verschiedenen Wirtschaftszweigen (vgl. Tabelle 1), darunter viele Architektur- und Ingenieurbüros. In den Ergebnissen unserer Analysen erhielten wir als höchst signifikanten Bestimmungsfaktor zur Bewertung von acht Strategien die Branche, d. h. die Selbsteinschätzung der befragten Unternehmen als Anbieter von Investitionsgütern, Konsumgütern oder Dienstleistungen.

\section{Zusammenfassung und Ausblick}

Borrmann et al. kamen zu dem Ergebnis, „daß die Internationalisierung für KMU in erster Linie ein endogener Lernprozeß ist, der sich exogen nicht beliebig beschleunigen läßt, und in dem zentrale Entwicklungsphasen nicht ohne weiteres übersprungen werden können “ (BORRMANN et al. 1996, S. 193). Diese Entwicklungs- 
phasen sind u. a. durch einen steigenden Kapitaleinsatz für internationale Aktivitäten gekennzeichnet. Oder, um es mit den Worten eines von uns befragten Außenwirtschaftsexperten in den Fachverbänden der gewerblichen Wirtschaft zu sagen: „Die Unternehmen müssen erst einmal ausreichend ,Schwungmasse für das internationale Geschäft sammeln."

Nach unseren Analyseergebnissen wird bei derartigen Aussagen zu wenig die Spezifik der jeweiligen Branche berücksichtigt. Zum einen sind für die Anbieter von Dienstleistungen, zumindest für Architektur- und Ingenieurbüros, kleinere Betriebsgrößen in Deutschland weitaus am häufigsten, wobei auch diese Unternehmen durchaus international aktiv sein können und wollen. Zum anderen sind, bedingt durch die spezifische Komplexität der Kundenwünsche im Business-to-BusinessMarketing (vgl. KLEINALTENKAMP u. PLINKE 1995) von Investitionsgütern und Dienstleistungen, auch jeweils andere Strategien für die Erschließung internationaler Märkte erforderlich.

Eine detaillierte Kenntnis dieser verschiedenen Strategien ist nach unserer Auffassung letztlich notwendig, um einerseits für Unternehmen verschiedener Branchen die jeweils optimale Strategie im internationalen Marketing zu finden, und andererseits Maßnahmen der Außenwirtschaftsförderung branchenspezifisch optimal konzipieren zu können. Für den Wirtschaftsraum Berlin-Brandenburg sehen wir aus den Ergebnissen unserer Analyse zwei Ansatzpunkte, um den Handel dieser Region mit asiatischen Partnern zu intensivieren:

1. Für die Anbieter von Konsumguitern und Investitionsgütern werden weitaus mehr Kontakte zu jenen Handelshäusern und -kommissionären benötigt, welche auf asiatischen Märkten bereits etabliert sind und die Produkte von brandenburgischen oder Berliner Unternehmen rasch auf den Markt bringen können.

2. Asiatische Partner für den Technologietransfer und das Kontraktmanagement benötigen insbesondere die Anbieter von Dienstleistungen. Für die Gewinnung asiatischer Kooperationspartner scheinen uns dabei Kontakte zu dortigen Fachverbänden und lokalen Ingenieurbüros besonders geeignet.

Die kleinen und mittleren Unternehmen der Region können bei der Realisierung der genannten Strategien zur Erschließung asiatischer Märkte durch die Fachverbände der gewerblichen Wirtschaft, die Geschäftsbanken sowie auch durch die Fachhochschulen und Universitäten unterstützt werden. Die Realisierung von kapitalintensiveren Strategien, beispielsweise die Eröffnung eines eigenen Vertriebsbüros in Beijing, würde dagegen eine verbesserte Eigenkapitalbasis und eine höhere Kreditwürdigkeit der Unternehmen erfordern. Die hierfür notwendigen steuer-, tarif- und wirtschaftspolitischen Maßnahmen liegen allerdings nur zu einem geringen Teil in der Kompetenz der beiden Bundesländer.

Wieweit - neben den von uns untersuchten Bestimmungsfaktoren - soziodemographische und psychographische Faktoren der Geschäftsfuihrer bzw. Inhaber der Unternehmen die Wahl der Strategie beeinflußen, kann mit dem bisher gesammelten Datenmaterial nicht analysiert werden. In den Längsschnittanalysen anderer Autoren konnte ein unterschiedlich hoher Einfluß der soziodemographischen und psychographischen Faktoren auf Exportumsatz, Exportquote und Exportrendite nachgewiesen werden (vgl. HOLZMÜLLER, KÖGLMAYR S. 133 sowie MÜLLER, S. 351). Je nach Definition des Erfolgskriteriums betrug der Anteil der psychographischen und soziodemographischen Faktoren an der regressionsanalytisch erklärten Varianz zwischen 13,7 und 51,8 Prozent. Diese Faktoren treten in einer Längsschnittanalyse deutlicher hervor, während eine Querschnittsanalyse (als einmalige Erhebung zum Zeitpunkt X) stärker von zufälligen Erfolgen oder Mißerfolgen der befragten Unternehmen im internationalen Geschäft beeinflußt wird.

Um so mehr halten wir es für notwendig, den internationalen Markterfolg gerade von jungen Kleinunternehmen verschiedener Branchen in der Region Brandenburg und Berlin kontinuierlich zu evaluieren, um über die Analyse von Erfolg und Mißerfolg letztlich zu empirisch gesicherten Erkenntnissen, validen Prognosen und praxisrelevanten Konzepten für Unternehmensführung und Wirtschaftspolitik zu gelangen.

\section{Tabellenverzeichnis}

Nr. Inhalt

Seite

1 Wirtschaftszweigstruktur Gesamtheit der 269 Unternehmen

2 Verteilung der Unternehmen nach Branchen (disjunkte Gruppierung)

3 Kundentypen der Unternehmen

4 Vermarktungsformen der Unternehmen

5 Übersicht über die von den Unternehmen zu bewertenden Strategien

6 Strategien - sonstige Nennungen

7 Verteilung der Anzahl der je Unternehmen bewerteten Strategien

8 Bewertung der Strategien nach Punktwerten

9 Eigenwerte der Faktoren und ihr Anteil an der Gesamtstreuung (Hauptkomponentenmethode)

10 Kommunalitäten für die acht Strategievariablen bei drei Faktoren (Hauptkomponentenmethode)

11 Sortierte Faktorladungsmatrix für drei Faktoren (Hauptkomponentenmethode) nach der VarimaxRotation

12 Durchschnittliche Bewertung der Strategien in ausgewählten Wirtschaftszweigen

13 Durchschnittliche Bewertung der Strategien in den disjunkt gruppierten Branchen

14 Ausgewählte Maßzahlen der Chi-Quadrat-Unabhängigkeitstests für Branche mit Bewertung der Strategien 1 bis 8

15 Durchschnittliche Bewertung der Strategien in fünf Unternehmensgrößen-Klassen, gruppiert nach Mitarbeiterzahl

16 Durchschnittliche Bewertung der Strategien in fünf Klassen nach Jahresumsatz 1995

17 Mittelwertvektoren der Cluster und Anzahl der Unternehmen im Cluster

18 Vermutete Bestimmungsfaktoren für die Clusterzugehörigkeit

19 Signifikante Bestimmungsfaktoren für die Clusterzugehörigkeit in der ersten Erklärungsebene 


\section{Verzeichnis der Abbildungen}

\begin{tabular}{rlr} 
Nr. & Inhalt & Seite \\
\hline 1 & $\begin{array}{l}\text { Branchen der Unternehmen (originäre } \\
\text { Gruppierung) }\end{array}$ & 23 \\
2 & $\begin{array}{l}\text { Gruppierung von Strategien im internationalen } \\
\text { Marketing nach Kapitaleinsatz und Kontroll- }\end{array}$ \\
& $\begin{array}{l}\text { rechten } \\
3\end{array}$ & $\begin{array}{l}\text { Strategiebewertungsprofile für ausgewählte } \\
\text { Wirtschaftszweige }\end{array}$ \\
4 & $\begin{array}{l}\text { Strategiebewertungsprofile für disjunkt } \\
\text { gruppierte Branchen }\end{array}$ \\
5 Strategiebewertungsprofil im Vergleich der & 28 \\
& $\begin{array}{l}\text { Mitarbeiterzahlen } \\
6\end{array}$ & $\begin{array}{l}\text { Strategiebewertung im Vergleich der Jahres- } \\
\text { umsätze 1995 }\end{array}$ \\
7 & $\begin{array}{l}\text { Clusterzugehörigkeit und CHAID-ANALYSE der } \\
\text { Bestimmungsfaktoren }\end{array}$ & 30 \\
&
\end{tabular}

\section{Literaturverzeichnis}

Adamer, Manfred M./Kaindl, Günter (1994): Erfolgsgeheimnis von Markt- und Weltmarktführern - eine analyse der Erfolgsfaktoren von erfolgreichen Unternehmen des deutschsprachigen Raumes; München und Mering Backhaus, Klaus (1992): Investitionsgütermarketing; 3. Auflage, München

Backhaus/Erichson/Plinke/Weiber: Multivariate Analysemethoden - Eine anwendungsorientierte Einfuihrung. Springer-Verlag, 1996

Bamberger, Ingolf/Evers, Michael (1994): Internationalisierungsverhalten von Klein- und Mittelunternehmen; in Engelhard, Johann und Rehkugler, Heinz (Hg.) (1994): Strategien für nationale und internationale Märkte; Wiesbaden

Beutel, Reiner (1988): Unternehmensstrategien international tätiger mittelständischer Unternehmen; Frankfurt a. M. u. a.

BMWi Bundesministerium für Wirtschaft (1995): Wirtschaft in Zahlen '95; Bonn

BMWi Bundesministerium für Wirtschaft (1996a): Exportfibel - Wegweiser für kleine und mittlere Unternehmen; Bonn

BMWi Bundesministerium für Wirtschaft (1996b): Investitionschancen und Erfahrungen kleiner und mittlerer Unternehmen im asiatisch-pazifischen Raum; Bonn Borrmann, Axel/Holthus, Manfred/Menck, Karl-Wolfgang/ Schnatz, Bernd (1996): Kleine und mittlere deutsche Unternehmen in Asien - Investitionschancen und Erfahrungen; Baden-Baden 1996

Brosius, Felix (1997): SPSS CHAID - Statistische Datenanalyse für Segmentierungsmodelle und Database Marketing; International Thomson Publishing Eckstein, Peter (1996): Statistische Datenanalyse mit SPSS; Wiesbaden

Forschner, Dirk (1996): Die Fahrmeisterausbildung für die Shanghai Metro Corporation; unveröffentlichte Dissertation FU Berlin

Henning, Christine und Schiewe, Dirk (1997): Chancen und Barrieren von kleinen und mittleren Unternehmen aus Berlin und Brandenburg beim Eintritt auf den asiatischen Markt; Diplomarbeit an der FHTW Berlin
Holzmüller, Hartmut H. (1995): Konzeptionelle und methodische Probleme in der interkulturellen Management- und Marketingforschung; Stuttgart Jenner, Thomas (1994): Internationale Marktbearbeitung - Erfolgreiche Strategien für Konsumgüterhersteller; Wiesbaden 1994

Kulicke, Marianne (1993): Chancen und Risiken junger Technologieunternehmen; Heidelberg

Kleinaltenkamp, Michael und Plinke, Wulff (Hg.) (1995): Technischer Vertrieb; Berlin u. a.

Köglmayr, Hans-Georg (1990): Die Auslandsorientierung von Managern als strategischer Erfolgsfaktor; Berlin Müller, Stefan (1996): Auslandsorientierung als Zielsetzung der Personalentwicklung; in: „Psychologie interkulturellen Handelns", hrsg. von Thomas, Alexander; Stuttgart 1996

Mugler, Josef (1995): Betriebswirtschaftslehre der Kleinund Mittelbetriebe; 2 . Auflage, Wien und NewYork Sauer, Jörg Dieter (1991): Das Exportverhalten von Handwerksbetrieben - Erkenntnisse aus empirischen Untersuchungen in Niedersachsen; Göttingen

Stahr, Gunter (1993): Internationales Marketing; Ludwigshafen (Rhein)

Wonneberger, Bernd/Könecke, Hans/Weickert, Sven (1997a): Asien - Wachstumsmärkte für brandenburgischen Unternehmen?; in: Wissenschaftliche Beiträge, TFH Wildau, Heft 1/1997

\section{Verfasser}

\section{Prof. Dr. Bernd Wonneberger \\ Dipl.-Psych. Hans Könecke \\ Dr. Rudolf Swat}

Technische Fachhochschule Wildau

Institut für Internationales Marketing und

Unternehmensführung

Bahnhofstraße

15745 Wildau

Tel. +49 (0) $3375-508325$

Fax +49 (0) $3375-508388$ 